JOURNAL OF

FUNCTION SPACES AND APPLICATIONS

Volume 6, Number 2 (2008), 107-154 (c) 2008, Scientific Horizon http://www.jfsa.net

\title{
On anisotropic Triebel-Lizorkin type spaces, with applications to the study of pseudo-differential operators
}

\author{
Lasse Borup and Morten Nielsen \\ (Communicated by Hans Feichtinger)
}

2000 Mathematics Subject Classification. 42B35, 35S05, 42C15.

Keywords and phrases. Anisotropic Triebel-Lizorkin spaces, anisotropic Bosov spaces, atomic decomposition, pseudo-differential operators.

\footnotetext{
Abstract. A construction of Triebel-Lizorkin type spaces associated with flexible decompositions of the frequency space $\mathbb{R}^{d}$ is considered. The class of admissible frequency decompositions is generated by a one parameter group of (anisotropic) dilations on $\mathbb{R}^{d}$ and a suitable decomposition function. The decomposition function governs the structure of the decomposition of the frequency space, and for a very particular choice of decomposition function the spaces are reduced to classical (anisotropic) Triebel-Lizorkin spaces. An explicit atomic decomposition of the Triebel-Lizorkin type spaces is provided, and their interpolation properties are studied. As the main application, we consider Hörmander type classes of pseudo-differential operators adapted to the anisotropy and boundedness of such operators between corresponding Triebel-Lizorkin type spaces is proved.
} 


\section{Introduction}

Besov and Triebel-Lizorkin spaces are two closely related families of smoothness spaces on $\mathbb{R}^{d}$ with important applications in approximation theory and harmonic analysis. The spaces are built from a resolution of unity in frequency space localized on dyadic annuli $\left\{2^{j-1}<|\xi|<2^{j+1}\right\}$. It follows from the definition that the spaces are isotropic and consequently well suited for the study of boundedness properties of "isotropic" differential operators such as powers of the Laplacian. However, there are many interesting differential operators that are not isotropic in nature. For example, consider the heat operator $L$ given by

$$
L(u):=\frac{\partial u}{\partial t}-\sum_{j=1}^{d} \frac{\partial^{2} u}{\partial x_{j}^{2}},
$$

where we differentiate once along the $t$-direction but twice along all other axes. For the study of such operators, anisotropic versions of Besov and Triebel-Lizorkin spaces are often more suitable. Anisotropic version of these spaces can be obtained by considering a resolution of unity supported on "anisotropic" dyadic annuli given by some anisotropic quasi-distance. Anisotropic Besov spaces were introduced in [2] and anisotropic TriebelLizorkin spaces were considered in [38].

The main contribution of this paper (presented in Section 5) is to introduce a new general construction of anisotropic smoothness spaces of Triebel-Lizorkin type (henceforth abbr. as T-L type) defined on $\mathbb{R}^{d}$. As an application of the new spaces, we study boundedness properties of certain associated classes of pseudo-differential operators. The spaces correspond to flexible but structured decompositions of the frequency space $\mathbb{R}^{d}$. The decompositions are generated by a one-parameter group of dilations on $\mathbb{R}^{d}$ and a suitable decomposition function. The group of dilations incorporates the anisotropy of the construction while the decomposition function governs the general splitting of the frequency space. Classical isotropic and anisotropic T-L spaces are recovered by a particular choice of decomposition function. As the main application of the new spaces, we consider mapping properties of pseudo-differential operators on the T$\mathrm{L}$ type spaces. We show that certain anisotropic Hörmander classes of symbols induce pseudo-differential operators that are bounded on the T-L type spaces. Tight frames for $L_{2}\left(\mathbb{R}^{d}\right)$ that gives a stable decomposition of the T-L type spaces are also considered. It is possible to use the frame to obtain sparse discrete representations of pseudo-differential operators. 
Several authors have built function spaces by considering a resolution of unity in frequency space. Feichtinger introduced the family of modulation spaces [16] which correspond to a uniform decomposition of frequency space. Modulation and Besov spaces are special cases of a very general construction of decomposition spaces introduced by Feichtinger and Gröbner [17] and Feichtinger [14]. Gröbner [24] used the decomposition space methods in [17] to define the so-called $\alpha$-modulation spaces as a family of intermediate spaces between modulation and Besov spaces. Group theoretical constructions of function spaces, including smoothness spaces, have been studied by Feichtinger and Gröchenig [15, 18, 19, 20, 25]. Frazier and Jawerth constructed frames (their so-called $\varphi$-transform) for Besov and T-L spaces in $[22,21]$. Their results were generalized recently by Bownik and Ho to the anisotropic case $[9,8]$. The present authors studied frame decomposition of Besov type spaces in [6].

One important application of Besov and T-L spaces is to the study of partial differential equations and to differential operators. Pseudodifferential operators (PDOs) on Besov and T-L spaces have been studied by many authors. For example, the Besov case was considered by Gibbons [23] and Bourdaud [7], while the T-L case was studied by Päivärinta [29] and Bui [10]. The anisotropic case was considered by Yamazaki $[40,39]$. PDOs have also been studied on spaces of Besov type based on non-dyadic frequency splittings. In particular, boundedness of such operators on modulation spaces has been considered by many authors, see e.g. $[12,13,26,28,31,34,35]$ and references herein. The authors studied PDOs on $\alpha$-modulation spaces in $[3,4]$.

The outline of the paper is as follows. Sections 2 to 4 contain the technical machinery needed to define the T-L spaces. More precisely, in section 2 we consider a homogeneous type structure on $\mathbb{R}^{d}$ with a quasi-distance induced by a one-parameter family of dilations. The infinitesimal generator $A$ of this group defines the anisotropic nature of the quasi-distance. The balls associated with the quasi-distance from Section 2 are not (in general) Euclidean balls and in Section 3 we introduce maximal functions compatible with the quasi-distance. The most important tools to study T-L type spaces are vector-valued estimates for the Hardy-Littlewood maximal function and for certain derived maximal functions. We develop versions of the maximal functions compatible with the anisotropic dilation and flexible splitting of the frequency space in Section 3. In Section 4 we introduce a family of structured splittings of the frequency spaces obtained by applying a countable family of affine transformations to a fixed compact set. The main contribution of the paper can be found in Section 5 where we define define a new family of T-L type spaces relative to a suitable resolution of the identity. It is proved that the spaces defined are quasi-Banach spaces independent of 
the particular resolution of identity. In Section 6 we introduce a tight frame for $L_{2}\left(\mathbb{R}^{d}\right)$ compatible with the frequency cover considered in Section 4. The frame gives an atomic decomposition of the T-L type spaces. Section 7 contains our main application of the new T-L spaces to a study of pseudodifferential operators. It is shown that for (anisotropic) Hörmander classes of symbols, the corresponding operators extend to bounded operators on suitable T-L type spaces. We conclude the paper in Section 8 by considering several concrete examples of T-L type spaces and some pseudo-differential operators on such spaces. Appendix A contains some of the more technical proofs.

Let us summarize some of the notation used throughout this paper. We let $\mathcal{F}(f)(\xi):=(2 \pi)^{-d / 2} \int_{\mathbb{R}^{d}} f(x) e^{-i x \cdot \xi} d x, f \in L_{1}\left(\mathbb{R}^{d}\right)$, denote the Fourier transform. By $F \asymp G$ we mean that there exist two constants $0<C_{1} \leq C_{2}<\infty$, depending only on "allowable" parameters, such that $C_{1} F \leq G \leq C_{2} F$. For two quasi-normed spaces $A$ and $B, A \hookrightarrow B$ means that $A \subset B$ and there exists a constant $C$ such that $\|f\|_{B} \leq C\|f\|_{A}$ for all

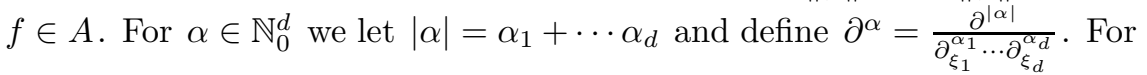
$0<p, q \leq \infty$, and a sequence $f=\left\{f_{j}\right\}_{j \in \mathbb{N}}$ of $L_{p}\left(\mathbb{R}^{d}\right)$ functions, we define the norm

$$
\|f\|_{L_{p}\left(\ell_{q}\right)}:=\left\|\left(\sum_{j \in \mathbb{N}}\left|f_{j}\right|^{q}\right)^{1 / q}\right\|_{L_{p}\left(\mathbb{R}^{d}\right)} .
$$

Where there is no risk of ambiguity we will abuse notation and write $\left\|f_{k}\right\|_{L_{p}\left(\ell_{q}\right)}$ instead of $\left\|\left\{f_{k}\right\}_{k}\right\|_{L_{p}\left(\ell_{q}\right)}$. Similarly, for $f=\left\{f_{j}\right\}_{j \in \mathbb{N}}$, we define

$$
\|f\|_{\ell_{q}\left(L_{p}\right)}:=\left(\sum_{j \in \mathbb{N}}\left\|f_{j}\right\|_{L_{p}}^{q}\right)^{1 / q} .
$$

Finally, for $\varphi \in \mathcal{S}^{\prime}\left(\mathbb{R}^{d}\right)$ and $f \in \mathcal{S}\left(\mathbb{R}^{d}\right)$ we let

$$
\varphi(D) f:=\mathcal{F}^{-1}(\varphi \mathcal{F} f)
$$

\section{A homogeneous type structure on $\mathbb{R}^{d}$}

In this section we define a homogeneous type space on $\mathbb{R}^{d}$ associated with a quasi-distance induced by a one-parameter group of dilations. We will use the quasi-distance in Section 4 to generate compatible coverings of the frequency space $\mathbb{R}^{d}$.

Let $|\cdot|$ denote the Euclidean norm on $\mathbb{R}^{d}$ induced by the inner product $(\cdot, \cdot)$. Suppose $A$ is a real $d \times d$ matrix with eigenvalues having positive real parts. For $t>0$ define the group of dilations $\delta_{t}: \mathbb{R}^{d} \rightarrow \mathbb{R}^{d}$ by 
$\delta_{t}=\exp (A \ln t)$. The matrix $A$ will be kept fixed throughout the paper. Let $\nu=\operatorname{trace}(A)$. The following properties are well-known (see [33])

- $\delta_{t s}=\delta_{t} \delta_{s}$, for $s, t>0$

- $\delta_{1}=I d$ (identity on $\mathbb{R}^{d}$ )

- $\delta_{t} \xi$ is jointly continuous in $t$ and $\xi$, and $\delta_{t} \xi \rightarrow 0$ as $t \rightarrow 0^{+}$

- $\left|\delta_{t}\right|:=\operatorname{det}\left(\delta_{t}\right)=t^{\nu}$

- There exist positive constants $c_{1}, c_{2}, \alpha_{1}$ and $\alpha_{2}$ such that for $\xi \in \mathbb{R}^{d}$,

$$
c_{1} \min \left\{t^{\alpha_{1}}, t^{\alpha_{2}}\right\}|\xi| \leq\left|\delta_{t} \xi\right| \leq c_{2} \max \left\{t^{\alpha_{1}}, t^{\alpha_{2}}\right\}|\xi| .
$$

Let $\sigma(A)$ denote the spectrum of $A$. Then we can choose $\alpha_{1}$ and $\alpha_{2}$ in (2.1) as any positive numbers satisfying

$$
\alpha_{1}<\min _{\lambda \in \sigma(A)} \operatorname{Re}(\lambda) \leq \max _{\lambda \in \sigma(A)} \operatorname{Re}(\lambda)<\alpha_{2},
$$

which can be seen by putting $A$ in Jordan canonical form. In the special case where $A$ is normal (and $A$ can be diagonalized over $\mathbb{C}$ ), $(2.2)$ can be relaxed allowing equality. When we use the symbols $\nu, \alpha_{1}$, and $\alpha_{2}$ in the sequel, we refer to the constants above.

According to [33, Proposition 1.7] there exists a strictly positive symmetric matrix $P$ such that for all $\xi \in \mathbb{R}^{d}$,

$$
\left[\delta_{t} \xi\right]_{P}:=\left(P \delta_{t} \xi, \delta_{t} \xi\right)^{1 / 2}
$$

is a strictly increasing function of $t$.

Definition 2.1. We define the function $|\cdot|_{A}: \mathbb{R}^{d} \rightarrow[0, \infty)$ by letting $|0|_{A}:=0$, and for $\xi \in \mathbb{R}^{d} \backslash\{0\}$ we define $|\xi|_{A}$ to be the unique solution $t$ to the equation $\left[\delta_{1 / t} \xi\right]_{P}=1$.

It is known that (see [33])

(1) $|\cdot|_{A} \in C^{\infty}\left(\mathbb{R}^{d} \backslash\{0\}\right)$

(2) There exists a constant $c$ such that

$$
|\xi+\zeta|_{A} \leq c\left(|\xi|_{A}+|\zeta|_{A}\right), \quad \forall \xi, \zeta \in \mathbb{R}^{d}
$$

(3) $\left|\delta_{t} \xi\right|_{A}=t|\xi|_{A}, t>0$.

(4) We have for $\xi \in \mathbb{R}^{d}$,

$$
c_{1} \min \left\{|\xi|_{A}^{\alpha_{1}},|\xi|_{A}^{\alpha_{2}}\right\} \leq|\xi| \leq c_{2} \max \left\{|\xi|_{A}^{\alpha_{1}},|\xi|_{A}^{\alpha_{2}}\right\} .
$$


(5) We can coordinatize $\mathbb{R}^{d}$ by $\rho$ and $\omega$ where $\rho=|\xi|_{A}$ and $\omega=\delta_{\rho}^{-1} \xi$. Then the volume element in $\mathbb{R}^{d}$ is

$$
d \xi=\rho^{\nu-1} d \omega d \rho,
$$

where $d \omega$ is the induced $C^{\infty}$ measure on the ellipsoid $\mathcal{S}:=\{\omega \in$ $\left.\mathbb{R}^{d}:[\omega]_{P}=1\right\}$.

Example 2.2. For $A=\operatorname{diag}\left(\beta_{1}, \beta_{2}, \ldots, \beta_{d}\right), \beta_{i}>0$, we have $\delta_{t}=$ $\operatorname{diag}\left(t^{\beta_{1}}, t^{\beta_{2}}, \ldots, t^{\beta_{d}}\right)$. In this case, one can check that

$$
|\xi|_{A} \asymp \sum_{j=1}^{d}\left|\xi_{j}\right|^{1 / \beta_{j}}, \quad \xi \in \mathbb{R}^{d} .
$$

The special choice, $A_{\text {iso }}=\operatorname{diag}(1,1, \ldots, 1)$ corresponds to the usual isotropic dilation and the corresponding distance $|\cdot|_{A_{\text {iso }}}$ is the Euclidean norm.

We need an extension of the quantity $\langle\xi\rangle:=\left(1+|\xi|^{2}\right)^{1 / 2}$ to the nonisotropic setting. Let $\tilde{A}$ be the $(d+1) \times(d+1)$ matrix given by

$$
\left[\begin{array}{ll}
1 & \mathbf{0} \\
\mathbf{0} & A
\end{array}\right]
$$

and define $D_{t}=\exp (\tilde{A} \ln t)$. For $(\zeta, \xi) \in \mathbb{R} \times \mathbb{R}^{d}$, we have $D_{t}(\zeta, \xi)=$ $\left(t \zeta, \delta_{t} \xi\right)$. We let $|(\zeta, \xi)|_{\tilde{A}}$ be the unique solution $t$ to $\left[\left[D_{1 / t}(\zeta, \xi)\right]\right]_{P}=1$, where $[[(\zeta, \xi)]]_{P}:=\left(\zeta^{2}+[\xi]_{P}^{2}\right)^{1 / 2}$. Notice that $|(1,0)|_{\tilde{A}}=1$ and $|(0, \xi)|_{\tilde{A}}=$ $|\xi|_{A}$. For $\xi \in \mathbb{R}^{d}$, we define the bracket $\langle\xi\rangle_{A}:=|(1, \xi)|_{\tilde{A}}$. The following lemma lists some properties of $\langle\cdot\rangle_{A}$.

Lemma 2.3. There exist constants $c, c^{\prime}$ such that for $\xi, \zeta \in \mathbb{R}^{d}$

a) $\langle\xi\rangle_{A} \geq 1,\langle\xi\rangle_{A} \asymp\left(1+|\xi|_{A}\right)$,

b) $\langle\xi+\zeta\rangle_{A} \leq c\left(\langle\xi\rangle_{A}+\langle\zeta\rangle_{A}\right)$,

c) $\left\langle\delta_{t} \xi\right\rangle_{A} \leq t\langle\xi\rangle_{A}$, if $t \geq 1$, and $\left\langle\delta_{t} \xi\right\rangle_{A} \leq 2 c \frac{t}{a}\langle\xi\rangle_{A}$, if $1>t \geq a>0$,

d) $\langle\xi+\zeta\rangle_{A} \leq c^{\prime}\langle\xi\rangle_{A}\langle\zeta\rangle_{A}$,

e) $\left|\partial^{\beta}\langle\xi\rangle_{A}^{s}\right| \leq C_{\beta}\langle\xi\rangle_{A}^{s-\alpha_{1}|\beta|}$, for all $\beta \in \mathbb{N}_{0}^{d}$.

Proof. We have $\langle\xi\rangle_{A}=|(1, \xi)|_{\tilde{A}} \geq|(1,0)|_{\tilde{A}}=1$. Moreover,

$$
\langle\xi\rangle_{A}=|(1, \xi)|_{\tilde{A}} \leq c\left(|(1,0)|_{\tilde{A}}+|(0, \xi)|_{\tilde{A}}\right)=c\left(1+|\xi|_{A}\right) .
$$

Clearly, $|\xi|_{A} \leq\langle\xi\rangle_{A}$ and $\langle\xi\rangle_{A} \geq 1$ so $2\langle\xi\rangle_{A} \geq\left(1+|\xi|_{A}\right)$. For b) we have $\langle\xi+\zeta\rangle_{A}:=|(1, \xi+\zeta)|_{\tilde{A}} \leq c\left(|(1, \xi)|_{\tilde{A}}+|(0, \zeta)|_{\tilde{A}}\right)=c\left(\langle\xi\rangle_{A}+|\zeta|_{A}\right) \leq c\left(\langle\xi\rangle_{A}+\langle\zeta\rangle_{A}\right)$, 
for the constant $c$ associated with the quasi-distance $|\cdot|_{\tilde{A}}$. Claim c), in the case $a \geq 1$, follows from

$$
\left\langle\delta_{t} \xi\right\rangle_{A}=\left|\left(1, \delta_{t} \xi\right)\right|_{\tilde{A}} \leq\left|\left(t, \delta_{t} \xi\right)\right|_{\tilde{A}}=\left|D_{t}(1, \xi)\right|_{\tilde{A}}=t\langle\xi\rangle_{A} .
$$

For $0<a<1$ we have

$$
\left\langle\delta_{t} \xi\right\rangle_{A} \leq c\left(1+\left|\delta_{t} \xi\right|_{A}\right)=c t\left(1 / t+|\xi|_{A}\right) \leq c \frac{t}{a}\left(1+|\xi|_{A}\right) \leq 2 c \frac{t}{a}\langle\xi\rangle_{A} .
$$

To prove d) suppose $u=\langle\xi\rangle_{A}$ and $v=\langle\zeta\rangle_{A}$. Let $c^{\prime} \geq 1$ be a constant such that $\left[\left[D_{1 / c^{\prime}} \eta\right]\right]_{P} \leq 1 / 2$ whenever $[[\eta]]_{P}=1, \eta \in \mathbb{R}^{d+1}$ (the existence of $c^{\prime}$ follows from (2.3)). We have $u, v \geq 1$ so

$$
\begin{aligned}
{\left[\left[D_{1 /\left(c^{\prime} u v\right)}(1, \xi+\zeta)\right]\right]_{P} } & \leq\left[\left[D_{1 / c^{\prime}} D_{1 / u}(1, \xi)\right]\right]_{P}+\left[\left[D_{1 / c^{\prime}} D_{1 / v}(0, \zeta)\right]\right]_{P} \\
& \leq\left[\left[D_{1 / c^{\prime}} D_{1 / u}(1, \xi)\right]\right]_{P}+\left[\left[D_{1 / c^{\prime}} D_{1 / v}(1, \zeta)\right]\right]_{P} \leq 1 .
\end{aligned}
$$

It follows that $|(1, \xi+\zeta)|_{\tilde{A}} \leq c^{\prime} u v$ so we obtain $\langle\xi+\zeta\rangle_{A} \leq c^{\prime}\langle\xi\rangle_{A}\langle\zeta\rangle_{A}$. Finally, e) follows from homogeneity of $|\cdot|_{\tilde{A}}$.

Remark 2.4. Notice that Lemma 2.3 has the following implications for a function $\eta$ on $\mathbb{R}^{d}$.

- $|\eta(\xi)| \leq C\langle\xi\rangle_{A}^{-M} \Longrightarrow|\eta(\xi)| \leq C^{\prime}(1+|\xi|)^{-M \alpha_{1}}$

- $|\eta(\xi)| \leq C(1+|\xi|)^{-N} \Longrightarrow|\eta(\xi)| \leq C^{\prime}\langle\xi\rangle_{A}^{-N / \alpha_{2}}$.

- Suppose $|\eta(\xi)| \leq C\langle\xi\rangle_{A}^{-\nu-\varepsilon}$ for $\xi \in \mathbb{R}^{d}$ for some $\varepsilon>0$. Then

$$
\int_{\mathbb{R}^{d}}|\eta(\xi)| d \xi \leq \int_{0}^{\infty} \rho^{\nu-1} \int_{\mathcal{S}}\left\langle\delta_{\rho} \omega\right\rangle_{A}^{-\nu-\varepsilon} d \omega d \rho \leq C \int_{0}^{\infty} \rho^{\nu-1} \rho^{-\nu-\varepsilon} d \rho<\infty .
$$

Finally, we define the balls $\mathcal{B}_{A}(\xi, r):=\left\{\zeta \in \mathbb{R}^{d}:|\xi-\zeta|_{A}<r\right\}$. It is easy to check that $\left|\mathcal{B}_{A}(\xi, r)\right|=r^{\nu} \omega_{d}^{A}$, with $\omega_{d}^{A}:=\left|\mathcal{B}_{A}(0,1)\right|$, so $\left(\mathbb{R}^{d},|\cdot|_{A}, d \xi\right)$ is a space of homogeneous type with homogeneous dimension $\nu$.

The ball $\mathcal{B}_{A}(\xi, r)=\delta_{r} \mathcal{B}_{A}(0,1)+\xi$ is convex. To see this, it suffices to verify that $\mathcal{B}_{A}(0,1)$ is convex. Let $\zeta, \eta \in \mathcal{B}_{A}(0,1)$ and put $u=$ $\max \left\{|\zeta|_{A},|\eta|_{A}\right\}$. For $0 \leq \theta \leq 1$, we have

$$
\left[\delta_{1 / u}(\theta \zeta+(1-\theta) \eta)\right]_{P} \leq \theta\left[\delta_{1 / u} \zeta\right]_{P}+(1-\theta)\left[\delta_{1 / u} \eta\right]_{P} \leq \theta+(1-\theta),
$$

so $|\theta \zeta+(1-\theta) \eta|_{A} \leq u<1$.

In the sequel, we let $B:=A^{\top}$ denote the transpose of $A$ wrt. the standard inner product on $\mathbb{R}^{d}$. Since the eigenvalues of $B$ have positive real parts $(\sigma(B)=\overline{\sigma(A)})$ we can repeat the above construction for the group $\delta_{t}^{\top}=\exp (B \ln t), t>0$. We let $|\cdot|_{B}$ and $\langle\cdot\rangle_{B}$ denote the quasi-distance and bracket corresponding to the group $\delta_{t}^{\top}$. Clearly, $|\cdot|_{B}$ and $\langle\cdot\rangle_{B}$ satisfy 
the same type of estimates as $|\cdot|_{A}$ and $\langle\cdot\rangle_{A}$. The balls associated with $|\cdot|_{B}$ are denoted $\mathcal{B}_{B}(x, r)$. We use the convention that $\delta_{t}$ acts on the frequency space while $\delta_{t}^{\top}$ acts in direct space.

\section{Some parabolic maximal function estimates}

The most useful tools to study T-L spaces on $\mathbb{R}^{d}$ are different types of maximal functions. In this section we state and prove some results on parabolic maximal functions adapted to the non-isotropic distances $|\cdot|_{A}$ and $|\cdot|_{B}$. Parabolic maximal functions are special cases of the general theory for maximal functions developed in [32, Chapters I\&II], see also [11].

For $0<r<\infty$, the parabolic maximal function of Hardy-Littlewood type is defined by

$$
M_{r}^{B} u(x):=\sup _{t>0}\left(\frac{1}{\omega_{d}^{B} \cdot t^{\nu}} \int_{\mathcal{B}_{B}(x, t)}|u(y)|^{r} d y\right)^{1 / r}, \quad u \in L_{r, \text { loc }}\left(\mathbb{R}^{d}\right),
$$

with $\omega_{d}^{B}:=\left|\mathcal{B}_{B}(0,1)\right|$. We use the notation $M^{B} u(x):=M_{1}^{B} u(x)$ in the sequel.

It is known (see [32, Chapters I\&II]) that the following extremely useful vector-valued Fefferman-Stein maximal inequality holds

$$
\left\|\left\{M_{r}^{B} f_{j}\right\}\right\|_{L_{p}\left(\ell_{q}\right)} \leq C_{B}\left\|\left\{f_{j}\right\}\right\|_{L_{p}\left(\ell_{q}\right)}
$$

in this setting for $r<q \leq \infty$ and $r<p<\infty, C_{B}:=C_{B}(r, p, q)$.

The following lemma gives us a pointwise estimate by maximal functions for convolutions with an approximation to the identity. For $\varphi \in L_{1}\left(\mathbb{R}^{d}\right)$, we define $\varphi_{\varepsilon}(x):=\left|\delta_{\varepsilon}\right|^{-1} \varphi\left(\delta_{1 / \varepsilon}^{\top} x\right)=\varepsilon^{-\nu} \varphi\left(\delta_{1 / \varepsilon}^{\top} x\right)$ for $\varepsilon>0$. Notice that $\widehat{\varphi_{\varepsilon}}(\xi)=\hat{\varphi}\left(\delta_{\varepsilon} \xi\right)$.

Lemma 3.1. Let $k:[0, \infty) \rightarrow[0, \infty)$ be a decreasing function, with $K(x)=k\left(|x|_{B}\right)$ an integrable function. Suppose $\varphi \in L_{1}\left(\mathbb{R}^{d}\right)$ satisfies $|\varphi(x)| \leq K(x)$. Then for $f \in L_{p}\left(\mathbb{R}^{d}\right), 1 \leq p \leq \infty$,

$$
\left|f * \varphi_{\varepsilon}(x)\right| \leq\|K\|_{L_{1}\left(\mathbb{R}^{d}\right)} M^{B} f(x), \quad \forall x \in \mathbb{R}^{d}, \varepsilon>0 .
$$

Proof. Let us first simplify the problem. Let $\tau_{h}$ be translation by $h \in \mathbb{R}^{d}$. Notice that $\tau_{h}\left(f * \varphi_{\varepsilon}\right)=\left(\tau_{h} f\right) * \varphi_{\varepsilon}$, and $\tau_{h} M^{B} f=M^{B}\left(\tau_{h} f\right)$ so it suffices to verify (3.2) at $x=0$. Also, the problem is dilation invariant with respect to $\varphi$ so we may take $\varepsilon=1$. Hence, we only need to estimate $|f * \varphi(0)| \leq(|f| * K)(0)$. Let us estimate $(|f| * K)(0)$ in the simple case where $K$ is of the form $\sum_{j=1}^{N} a_{j} \chi_{\mathcal{B}_{B}\left(0, r_{j}\right)}$, with each $a_{j}$ a positive constant. Then 
since $\sum a_{j}\left|\chi_{\mathcal{B}_{B}\left(0, r_{j}\right)}\right|=\|K\|_{L_{1}}$ and $\left(|f| * \chi_{\mathcal{B}_{B}\left(0, r_{j}\right)}\right)(0) \leq\left|\chi_{\mathcal{B}_{B}\left(0, r_{j}\right)}\right| M^{B} f(0)$, (3.2) follows in this special case. For the general case, we notice that any non-negative, $|\cdot|_{B}$-symmetric and $|\cdot|_{B}$-decreasing integrable function $K$ can be approximated from below by such finite sums.

The following easy corollary will be used later to study multipliers on $L_{p}\left(\ell_{q}\right)$.

Corollary 3.2. Let $k_{n}:[0, \infty) \rightarrow[0, \infty), n \in \mathbb{N}$, be a sequence of decreasing continuous functions and suppose $K_{n}(x)=k_{n}\left(|x|_{B}\right), x \in \mathbb{R}^{d}$, is a bounded sequence in $L_{1}\left(\mathbb{R}^{d}\right)$. Assume $\left\{\psi_{n}\right\}_{n \in \mathbb{N}}$ is a sequence of $L_{1}\left(\mathbb{R}^{d}\right)$ functions with $\left|\psi_{n}(x)\right| \leq K_{n}(x)$. Then there exists a constant $C<\infty$ such that for $\left\{f_{n}\right\}_{n} \in L_{p}\left(\ell_{q}\right), 1<p<\infty, 1<q \leq \infty$,

$$
\left\|\left\{\psi_{n} * f_{n}\right\}\right\|_{L_{p}\left(\ell_{q}\right)} \leq C \sup _{m}\left\|K_{m}\right\|_{L_{1}} \cdot\left\|\left\{f_{n}\right\}\right\|_{L_{p}\left(\ell_{q}\right)} .
$$

Proof. We have the uniform pointwise estimate $\left|\psi_{n} * f_{n}(x)\right| \leq$ $\sup _{m}\left\|K_{m}\right\|_{L_{1}} M^{B} f_{n}(x)$. Hence, by the Fefferman-Stein inequality,

$\left\|\left\{\psi_{n} * f_{n}\right\}\right\|_{L_{p}\left(\ell_{q}\right)} \leq \sup _{m}\left\|K_{m}\right\|_{L_{1}}\left\|\left\{M^{B} f_{n}\right\}\right\|_{L_{p}\left(\ell_{q}\right)} \leq C \sup _{m}\left\|K_{m}\right\|_{L_{1}}\left\|\left\{f_{n}\right\}\right\|_{L_{p}\left(\ell_{q}\right)}$.

We introduce the following type of maximal function of Fefferman-Stein type as a tool to prove Theorem 3.5 below. Let $u(x)$ be a continuous function on $\mathbb{R}^{d}$. We define

$$
u^{*}(a, R ; x):=\sup _{y \in \mathbb{R}^{d}}\langle y\rangle_{B}^{-a}\left|u\left(x-\delta_{R}^{-\top} y\right)\right|,
$$

with the compact notation $\delta_{R}^{-\top}:=\left(\delta_{R}^{-1}\right)^{\top}$.

It is clear that $u^{*}(a, R ; x)$ is finite whenever $u$ is bounded. However, for band-limited functions we can obtain a much more interesting estimate of $u^{*}(a, R ; x)$ in terms of the parabolic maximal function. The following result is adapted to our setup from the isotropic setting in [36, Theorem 1.3.1]. A detailed proof can be found in Appendix A.

Proposition 3.3. Suppose $r, R>0$. Then there exist a constant $C:=$ $C(R, r)$ such that for any function $u(x)$ on $\mathbb{R}^{d}$ with $\operatorname{supp}(\hat{u}) \subset \mathcal{B}_{A}(0, R)$, we have

$$
u^{*}(\nu / r, R ; x) \leq C M_{r}^{B} u(x), \quad \forall x \in \mathbb{R}^{d} .
$$

Proposition 3.3 is even more useful when it is combined with the Fefferman-Stein maximal inequality. We have the following corollary, where 
for $\Omega=\left\{\Omega_{n}\right\}$ a sequence of compact subsets of $\mathbb{R}^{d}$, we let

$$
L_{p}^{\Omega}\left(\ell_{q}\right):=\left\{\left\{f_{n}\right\}_{n \in \mathbb{N}} \in L_{p}\left(\ell_{q}\right) \mid \operatorname{supp}\left(\hat{f}_{n}\right) \subseteq \Omega_{n}, \forall n\right\} .
$$

Corollary 3.4. Suppose $0<p<\infty$ and $0<q \leq \infty$, and let $\Omega=\left\{T_{k} \mathcal{C}\right\}_{k \in \mathbb{N}}$ be a sequence of compact subsets of $\mathbb{R}^{d}$ generated by a family $\left\{T_{k}=\delta_{t_{k}} \cdot+\xi_{k}\right\}_{k \in \mathbb{N}}$ of invertible affine transformations on $\mathbb{R}^{d}$, with $\mathcal{C}$ a fixed compact subset of $\mathbb{R}^{d}$. If $0<r<\min (p, q)$, then there exists a constant $K$ such that

$$
\left\|\left\{\sup _{z \in \mathbb{R}^{d}}\left\langle\delta_{t_{k}}^{\top} z\right\rangle_{B}^{-\nu / r}\left|f_{k}(\cdot-z)\right|\right\}\right\|_{L_{p}\left(\ell_{q}\right)} \leq K\left\|\left\{f_{k}\right\}\right\|_{L_{p}\left(\ell_{q}\right)},
$$

for all $f \in L_{p}^{\Omega}\left(\ell_{q}\right)$, where $f=\left\{f_{k}\right\}_{k \in \mathbb{N}}$.

Proof. Replacing $f_{k}(x)$ by $e^{-i x \cdot \xi_{k}} f_{k}(x)$, we may assume that

$$
\operatorname{supp}\left(\hat{f}_{k}\right) \subseteq \delta_{t_{k}} \mathcal{C} \subseteq \delta_{t_{k}} \mathcal{B}_{A}(0, R)=\mathcal{B}_{A}\left(0, t_{k} R\right)
$$

for some fixed $R<\infty$. By Proposition 3.3,

$$
\sup _{z \in \mathbb{R}^{d}}\left\langle\delta_{R t_{k}}^{\top} z\right\rangle_{A}^{-\nu / r}\left|f_{k}(\cdot-z)\right| \leq C M_{r}^{B}\left(f_{k}\right),
$$

and since $\left\langle\delta_{R t_{k}}^{\top} z\right\rangle_{B} \leq C R\left\langle\delta_{t_{k}} z\right\rangle_{B}$ for all $z \in \mathbb{R}^{d}$ and $R>0$,

$$
\sup _{z \in \mathbb{R}^{d}}\left\langle\delta_{t_{k}}^{\top} z\right\rangle_{B}^{-\nu / r}\left|f_{k}(\cdot-z)\right| \leq C R^{\nu / r} M_{r}^{B}\left(f_{k}\right) .
$$

Hence,

$$
\left\|\left\{\sup _{z \in \mathbb{R}^{d}}\left\langle\delta_{t_{k}}^{\top} z\right\rangle_{B}^{-\nu / r}\left|f_{k}(\cdot-z)\right|\right\}\right\|_{L_{p}\left(\ell_{q}\right)} \leq C^{\prime}\left\|\left\{M_{r}^{B} f_{k}\right\}\right\|_{L_{p}\left(\ell_{q}\right)} .
$$

We conclude using the Fefferman-Stein maximal inequality,

$$
\left\|\left\{\sup _{z \in \mathbb{R}^{d}}\left\langle\delta_{t_{k}}^{\top} z\right\rangle_{B}^{-\nu / r}\left|f_{k}(\cdot-z)\right|\right\}\right\|_{L_{p}\left(\ell_{q}\right)} \leq C^{\prime}\left\|M_{r}^{B} f_{k}\right\|_{L_{p}\left(\ell_{q}\right)} \leq K\left\|\left\{f_{k}\right\}\right\|_{L_{p}\left(\ell_{q}\right)} .
$$

We conclude this section by the following theorem on multipliers for vector-valued band-limited functions. The result is inspired by [36, Theorem 1.6.3], and we will use it in Section 5 to define T-L type spaces. For $s \in \mathbb{R}$ we let

$$
\|f\|_{H_{2}^{s}}:=\left(\int\left|\mathcal{F}^{-1} f(x)\right|^{2}\langle x\rangle_{B}^{2 s} d x\right)^{1 / 2}
$$


denote the (anisotropic) Sobolev space norm. Notice that for $f \in H_{2}^{s}, 0<$ $\varepsilon_{0} \leq t<\infty$, and $\xi_{k} \in \mathbb{R}^{d}$, we have $\left\|f\left(\delta_{t} \cdot+\xi_{k}\right)\right\|_{H_{2}^{s}} \leq C t^{s-\nu / 2}\|f\|_{H_{2}^{s}}<\infty$ with $C$ depending only on $\varepsilon_{0}$.

Theorem 3.5. Suppose $0<p<\infty$ and $0<q \leq \infty$. Let $\Omega=$ $\left\{T_{k} \mathcal{C}\right\}_{k \in \mathbb{N}}$ be a sequence of compact subsets of $\mathbb{R}^{d}$ generated by a family $\left\{T_{k}=\delta_{t_{k}} \cdot+\xi_{k}\right\}_{k \in \mathbb{N}}$ of invertible affine transformations, with $\mathcal{C}$ a fixed compact subset of $\mathbb{R}^{d}$. Assume $\left\{\psi_{j}\right\}_{j \in \mathbb{N}}$ is a sequence of functions satisfying $\psi_{j} \in H_{2}^{s}$ for some $s>\frac{\nu}{2}+\frac{\nu}{\min (p, q)}$. Then there exists a constant $C<\infty$ such that

$$
\left\|\left\{\psi_{k}(D) f_{k}\right\}\right\|_{L_{p}\left(\ell_{q}\right)} \leq C \sup _{j}\left\|\psi_{j}\left(T_{j} \cdot\right)\right\|_{H_{2}^{s}} \cdot\left\|\left\{f_{k}\right\}\right\|_{L_{p}\left(\ell_{q}\right)}
$$

for all $\left\{f_{k}\right\}_{k \in \mathbb{N}} \in L_{p}^{\Omega}\left(\ell_{q}\right)$.

Proof of Theorem 3.5. The proof relies on Corollary 3.4. In fact, since

$$
\left|\psi_{k}(D) f_{k}(x)\right| \leq \sup _{z \in \mathbb{R}^{d}} \frac{\left|\psi_{k}(D) f_{k}(x-z)\right|}{\left\langle\delta_{t_{k}}^{\top} z\right\rangle_{B}^{\frac{\nu}{r}}}
$$

pointwise a.e., the theorem follows by Corollary 3.4 if we can show that

$$
\sup _{z \in \mathbb{R}^{d}} \frac{\left|\psi_{k}(D) f_{k}(x-z)\right|}{\left\langle\delta_{t_{k}}^{\top} z\right\rangle_{B}^{\frac{\nu}{r}}} \leq C \sup _{z \in \mathbb{R}^{d}} \frac{\left|f_{k}(x-z)\right|}{\left\langle\delta_{t_{k}}^{\top} z\right\rangle_{B}^{\frac{\nu}{r}}} \cdot\left\|\psi_{k}\left(T_{k} \cdot\right)\right\|_{H_{2}^{s}}
$$

is valid a.e., when $s>\frac{\nu}{2}+\frac{\nu}{r}$.

It can be verified that $\check{\psi_{k}} \in L_{1}$, with $\check{\psi}_{k}$ the inverse Fourier transform of $\psi_{k}$. Thus $\psi_{k}(D) f_{k}$ can be rewritten as a convolution $\check{\psi}_{k} * f_{k}$ (up to a constant). In particular,

$$
\begin{aligned}
\left|\psi_{k}(D) f_{k}(x-z)\right| \leq & C \int_{\mathbb{R}^{d}}\left|\check{\psi}_{k}(x-z-y)\right| \cdot\left|f_{k}(y)\right| d y \\
\leq & C \sup _{u \in \mathbb{R}^{d}} \frac{\left|f_{k}(u)\right|}{\left\langle\delta_{t_{k}}^{\top}(x-u)\right\rangle_{B}^{\frac{\nu}{r}}} \\
& \times \int_{\mathbb{R}^{d}}\left|\check{\psi}_{k}(x-z-y)\right|\left\langle\delta_{t_{k}}^{\top}(x-y)\right\rangle_{B}^{\frac{\nu}{r}} d y .
\end{aligned}
$$

Recall that $\left\langle\delta_{t_{k}}^{\top}(x-y)\right\rangle_{B} \leq c^{\prime}\left\langle\delta_{t_{k}}^{\top}(x-z-y)\right\rangle_{B} \cdot\left\langle\delta_{t_{k}}^{\top} z\right\rangle_{B}$ (see Lemma 2.3). Using this in (3.6) and dividing by $\left\langle\delta_{t_{k}}^{\top} z\right\rangle_{B}^{\frac{\nu}{r}}$ gives

$$
\sup _{z \in \mathbb{R}^{d}} \frac{\left|\psi_{k}(D) f_{k}(x-z)\right|}{\left\langle\delta_{t_{k}}^{\top} z\right\rangle_{B}^{\frac{\nu}{r}}} \leq C \sup _{z \in \mathbb{R}^{d}} \frac{\left|f_{k}(x-z)\right|}{\left\langle\delta_{t_{k}}^{\top} z\right\rangle_{B}^{\frac{\nu}{r}}} \int_{\mathbb{R}^{d}}\left|\check{\psi}_{k}(y)\right|\left\langle\delta_{t_{k}}^{\top} y\right\rangle_{B}^{\frac{\nu}{r}} d y .
$$


Finally, using $\left|\check{\psi}_{k}(y)\right|=\left|\delta_{t_{k}}\right|\left|\left(\mathcal{F}^{-1} \psi_{k}\left(T_{k} \cdot\right)\right)\left(\delta_{t_{k}}^{\top} y\right)\right|$ and that $s>\frac{\nu}{r}+\frac{\nu}{2}$, we obtain

$$
\begin{aligned}
\int_{\mathbb{R}^{d}}\left|\check{\psi}_{k}(y)\right|\left\langle\delta_{t_{k}}^{\top} y\right\rangle_{B}^{\frac{\nu}{r}} d y & =\int_{\mathbb{R}^{d}}\left|\left(\mathcal{F}^{-1} \psi_{k}\left(T_{k} \cdot\right)\right)(y)\right|\langle y\rangle_{B}^{\frac{\nu}{r}} d y \\
& \leq C\left[\int_{\mathbb{R}^{d}}\left|\left(\mathcal{F}^{-1} \psi_{k}\left(T_{k} \cdot\right)\right)(y)\right|^{2}\langle y\rangle_{B}^{2 s} d y\right]^{\frac{1}{2}} \\
& =C\left\|\psi_{k}\left(T_{k} \cdot\right)\right\|_{H_{2}^{s}} .
\end{aligned}
$$

Thus, the inequality (3.5) holds true and the theorem follows by Corollary 3.4.

\section{Structured admissible coverings}

The purpose of this section is to provide smooth resolutions of the identity on $\mathbb{R}^{d}$ which will be used later in Section 5 to define T-L type smoothness spaces. By a resolution of the identity we mean a countable collection of nonnegative smooth functions $\left\{\varphi_{k}\right\}_{k}$ on $\mathbb{R}^{d}$ with $\sum_{k} \varphi_{k}=1$. Additionally, we also require some uniform control on each $\varphi_{k}$ that will be spelled out in Definition 4.8 below. The resolution of identity will induce a certain splitting of the frequency space $\mathbb{R}^{d}$ that again will determine the properties of the associated T-L type spaces. We therefore stride to construct the most flexible decompositions possible while retaining the uniform control. We begin by considering structured coverings of $\mathbb{R}^{d}$.

4.1. Admissible coverings and BAPUs. Here we study admissible coverings of $\mathbb{R}^{d}$, which we will consider as the frequency domain. The coverings will be used to introduce suitable partitions of unity on $\mathbb{R}^{d}$.

Definition 4.1. A set $\mathcal{Q}:=\left\{Q_{i}\right\}_{i \in \mathbb{N}}$ of measurable subsets $Q_{i} \subset \mathbb{R}^{d}$ is called an admissible covering if $\mathbb{R}^{d}=\cup_{i \in \mathbb{N}} Q_{i}$ and there exists $n_{0}<\infty$ such that $\#\left\{j \in \mathbb{N}: Q_{i} \cap Q_{j} \neq \emptyset\right\} \leq n_{0}$ for all $i \in \mathbb{N}$.

Let us introduce some notation needed to study properties of admissible coverings. Given an admissible covering $\left\{Q_{i}\right\}_{i \in \mathbb{N}}$ of $\mathbb{R}^{d}$ and a subset $J \subset \mathbb{N}$, we define

$$
\tilde{J}:=\left\{i \in \mathbb{N}: \exists j \in J \text { s.t. } Q_{i} \cap Q_{j} \neq \emptyset\right\} .
$$

Furthermore, let $\tilde{J}^{(0)}:=J$, and define inductively $\tilde{J}^{(k+1)}:=\widetilde{\tilde{J}^{(k)}}, k \geq 0$. We write $\tilde{i}^{(k)}:=\widetilde{\{i\}}{ }^{(k)}$ and $\tilde{i}:=\widetilde{\{i\}}$ for a singleton set. Notice that 
$\tilde{i}:=\left\{j \in \mathbb{N}: Q_{i} \cap Q_{j} \neq \emptyset\right\}$. Finally, we denote

$$
{\widetilde{Q_{i}}}^{(k)}:=\bigcup_{j \in \tilde{i}^{(k)}} Q_{j}
$$

Definition 4.2. Let $\mathcal{Q}=\left\{Q_{i}\right\}_{i \in \mathbb{N}}$ and $\mathcal{P}=\left\{P_{j}\right\}_{j \in \mathbb{N}}$ be two admissible coverings of $\mathbb{R}^{d}$. $\mathcal{Q}$ is called subordinate to $\mathcal{P}$ if for every $i \in \mathbb{N}$ there exists $j \in \mathbb{N}$ such that $Q_{i} \subseteq P_{j}$. $\mathcal{Q}$ is called almost subordinate to $\mathcal{P}$ (written $\mathcal{Q} \leq \mathcal{P})$ if there exists $k \in \mathbb{N}$ such that $\mathcal{Q}$ is subordinate to $\left\{\widetilde{P}_{j}^{(k)}\right\}_{j \in \mathbb{N}}$. If $\mathcal{Q} \leq \mathcal{P}$ and $\mathcal{P} \leq \mathcal{Q}$ the two coverings are called equivalent and we write $\mathcal{Q} \sim \mathcal{P}$.

In the generality we will consider below, there is a simplified definition of equivalence for admissible coverings proved in [17].

Theorem 4.3 ([17]). Let $\mathcal{Q}=\left\{Q_{i}\right\}_{i \in \mathbb{N}}$ and $\mathcal{P}=\left\{P_{j}\right\}_{j \in \mathbb{N}}$ be two admissible coverings of $\mathbb{R}^{d}$ both consisting of open arcwise connected subsets. Then $\mathcal{Q} \sim \mathcal{P}$ if and only if $\sup _{i \in \mathbb{N}} \# J(i)<\infty$ and $\sup _{j \in \mathbb{N}} \# I(j)<\infty$, where

$$
J(i):=\left\{j \in \mathbb{N} \mid Q_{i} \cap P_{j} \neq \emptyset\right\}, \quad I(j):=\left\{i \in \mathbb{N} \mid P_{j} \cap Q_{i} \neq \emptyset\right\} .
$$

We now turn to the construction of the admissible coverings of $\mathbb{R}^{d}$. To keep the construction geometrically simple and thus useful, we follow [17] and use a suitable collection of $|\cdot|_{A}$-balls to cover $\mathbb{R}^{d}$. Another simplification is that we choose the radius of a given ball in the cover as a suitable function of its center. The following class of regulation functions will be useful for that purpose.

Definition 4.4. A function $h: \mathbb{R}^{d} \rightarrow\left[\varepsilon_{0}, \infty\right)$ for $\varepsilon_{0}>0$ is called moderate if there exist constants $\rho_{0}, R_{0}$ such that $|\xi-\zeta|_{A} \leq \rho_{0} h(\xi)$ implies $R_{0}^{-1} \leq h(\zeta) / h(\xi) \leq R_{0}$.

The following lemma proved in [14] provides an abundance of moderate functions.

Lemma 4.5. Let $h: \mathbb{R}^{d} \rightarrow[1, \infty)$ be a weakly subadditive function, i.e., there exist a constant $C_{1}$ such that $h(\xi+\zeta) \leq C_{1}(h(\xi)+h(\zeta))$, for all $\xi, \zeta \in \mathbb{R}^{d}$. Assume furthermore that $h$ satisfies the growth condition

$$
h(\xi) \leq C\langle\xi\rangle_{A}, \quad \text { for all } \xi \in \mathbb{R}^{d} .
$$

Then there exists $\rho_{0}, R_{0}>0$ such that $h$ is moderate. In particular, suppose $s:[0, \infty) \rightarrow[1, \infty)$ is a non-decreasing function satisfying $s(2 t) \leq K_{s} s(t)$ and $s(t) \leq C_{s}(1+t)$ for all $t \geq 0$, then $h(\xi):=s\left(|\xi|_{A}\right)$ is weakly subadditive (and thus moderate). 
The following is a typical example of a moderate function which we will return to several times in the sequel.

Example 4.6. Let $0 \leq \alpha \leq 1$. Then $s(t)=(1+t)^{\alpha}$ is subadditive and consequently,

$$
h(\xi):=\left(1+|\xi|_{A}\right)^{\alpha} \asymp\langle\xi\rangle_{A}^{\alpha},
$$

is moderate.

Given a moderate function $h$, it is possible to construct a nice admissible covering by balls. The details are laid out in the following lemma.

Lemma 4.7. Given a moderate function $h$ with constants $R, \rho_{0}>0$, then

a) there exists a countable admissible covering $\mathcal{C}=\left\{\mathcal{B}_{A}\left(\xi_{j}, \rho h\left(\xi_{j}\right)\right)\right\}_{j \in \mathbb{N}}$ for $\rho<\rho_{0} / 2$, and there exists a constant $0<\rho^{\prime}<\rho$ such that the members of $\left\{\mathcal{B}_{A}\left(\xi_{j}, \rho^{\prime} h\left(\xi_{j}\right)\right)\right\}_{j \in \mathbb{N}}$ are pairwise disjoint.

b) Any two admissible coverings of the type considered in a) are equivalent in the sense of Definition 4.2.

Proof. We only give an outline of the proof. The covering can be constructed as follows.

- Put $\rho^{\prime}=\rho /\left(1+2 c R^{2}\right)$, with $c$ the constant from the quasi-triangle inequality for $|\cdot|_{A}$ and $R$ the constant associated with $h$. Pick a maximal pairwise disjoint collection $\left\{\mathcal{B}_{A}\left(\xi_{j}, \rho^{\prime} h\left(\xi_{j}\right)\right)\right\}_{j \in \mathbb{N}}$.

- Using the quasi-triangle inequality, $h$ is moderate, and the fact that

$$
\left|\mathcal{B}_{A}\left(\xi_{j}, \rho^{\prime} h\left(\xi_{j}\right)\right)\right| \asymp h\left(\xi_{j}\right)^{\nu}
$$

we can verify that $\mathcal{C}=\left\{\mathcal{B}_{A}\left(\xi_{j}, \rho h\left(\xi_{j}\right)\right)\right\}_{j \in \mathbb{N}}$ is an admissible covering of open convex subsets of $\mathbb{R}^{d}$.

That any two such coverings are equivalent can be verified by calling on Theorem 4.3. Let $\left\{\mathcal{B}_{A}\left(\xi_{j}, \rho_{1} h\left(\xi_{j}\right)\right)\right\}_{j \in \mathbb{N}}$ and $\left\{\mathcal{B}_{A}\left(\zeta_{i}, \rho_{2} h\left(\zeta_{i}\right)\right)\right\}_{i \in \mathbb{N}}$ be two such coverings. Suppose

$$
\mathcal{B}_{A}\left(\xi_{j_{0}}, \rho_{1} h\left(\xi_{j_{0}}\right)\right) \cap \mathcal{B}_{A}\left(\zeta_{i_{0}}, \rho_{2} h\left(\zeta_{i_{0}}\right)\right) \neq \emptyset .
$$

Using the moderation of $h$ one verifies that $h\left(\xi_{j_{0}}\right) \leq R^{2} h\left(\zeta_{i_{0}}\right)$ and that

$$
\mathcal{B}_{A}\left(\xi_{j_{0}}, \rho_{1} h\left(\xi_{j_{0}}\right)\right) \subseteq \mathcal{B}_{A}\left(\zeta_{i_{0}},\left(1+c R^{2}\right) \rho_{2} h\left(\zeta_{i_{0}}\right)\right) .
$$

Using that the balls in $\left\{\mathcal{B}_{A}\left(\xi_{j}, \rho^{\prime} h\left(\xi_{j}\right)\right)\right\}_{j \in \mathbb{N}}$ are pairwise disjoint together with the estimate

$$
\left|\mathcal{B}_{A}\left(\xi_{j_{0}}, \rho^{\prime} h\left(\xi_{j_{0}}\right)\right)\right| \asymp h\left(\xi_{j_{0}}\right)^{\nu} \asymp h\left(\zeta_{i_{0}}\right)^{\nu}
$$


one easily verifies that the "finite overlap" conditions of Theorem 4.3 are satisfied.

Notice that the covering $\mathcal{C}$ from Lemma 4.7 is generated by a family of invertible affine transformations applied to $\mathcal{B}_{A}(0, \rho)$ in the sense that

$$
\mathcal{B}_{A}\left(\xi_{j}, \rho h\left(\xi_{j}\right)\right)=T_{j} \mathcal{B}_{A}(0, \rho), \quad T_{j}:=\delta_{h\left(\xi_{j}\right)} \cdot+\xi_{j} .
$$

One can verify that $\left\{T_{k} \mathcal{B}_{A}(0,2 \rho)\right\}_{k}$ is an admissible covering using that the balls in the collection $\left\{\mathcal{B}_{A}\left(\xi_{j}, \rho^{\prime} h\left(\xi_{j}\right)\right)\right\}_{j \in \mathbb{N}}$ are pairwise disjoint. We call such a covering a structured admissible covering. An important property of the covering we will need is that whenever $\mathcal{B}_{A}\left(\xi_{j}, 2 \rho h\left(\xi_{j}\right)\right) \cap$ $\mathcal{B}_{A}\left(\xi_{k}, 2 \rho h\left(\xi_{k}\right)\right) \neq \emptyset$ then $h\left(\xi_{j}\right) \asymp h\left(\xi_{k}\right)$ uniformly in $j$ and $k$ which follows from the fact that $h$ is moderate and $2 \rho<\rho_{0}$. We deduce that there exists a uniform constant $K$ such that

$\left\|\delta_{h\left(\xi_{k}\right)}^{-1} \delta_{h\left(\xi_{j}\right)}\right\|_{\ell_{2}\left(\mathbb{R}^{d^{2}}\right)} \leq K \quad$ whenever $\mathcal{B}_{A}\left(\xi_{j}, 2 \rho h\left(\xi_{j}\right)\right) \cap \mathcal{B}_{A}\left(\xi_{k}, 2 \rho h\left(\xi_{k}\right)\right) \neq \emptyset$.

We are not really interested in the admissible covering $\mathcal{C}$ itself, but we will use it as a tool to generate partitions of unity that can be used to define T-L type spaces compatible with the covering $\mathcal{C}$. For technical reasons we require the partition of unity to satisfy the following.

Definition 4.8. Let $\mathcal{C}:=\left\{T_{k} \mathcal{B}_{A}(0, \rho)\right\}_{k \in \mathbb{N}}$ be an admissible covering of $\mathbb{R}^{d}$ of the type considered in Lemma 4.7. A corresponding bounded admissible partition of unity (BAPU) is a family of functions $\left\{\varphi_{k}\right\}_{k \in \mathbb{N}} \subset$ $\mathcal{S}\left(\mathbb{R}^{d}\right)$ satisfying

(a) $\operatorname{supp}\left(\varphi_{k}\right) \subset T_{k} \mathcal{B}_{A}(0,2 \rho), \quad k \in \mathbb{N}$,

(b) $\sum_{k \in \mathbb{N}} \varphi_{k}(\xi)=1, \quad \forall \xi \in \mathbb{R}^{d}$,

(c) $\sup _{k \in \mathbb{N}}\left\|\varphi_{k}\left(T_{k} \cdot\right)\right\|_{H_{2}^{s}}<\infty, \quad \forall s>0$.

A BAPU can easily be constructed for $\mathcal{C}$. Pick $\Phi \in C^{\infty}\left(\mathbb{R}^{d}\right)$ nonnegative with $\operatorname{supp}(\Phi) \subset \mathcal{B}_{A}(0,2 \rho)$ and $\Phi(\xi)=1$ for $\xi \in \mathcal{B}_{A}(0, \rho)$. Then we claim that

$$
\varphi_{j}(\xi):=\frac{\Phi\left(T_{j}^{-1} \xi\right)}{\sum_{k \in \mathbb{N}} \Phi\left(T_{k}^{-1} \xi\right)}
$$

defines an associated BAPU. Only property (c) in Definition 4.8 is not obvious. There exists a constant $K<\infty$ such that $1 \leq \sum_{k} \Phi\left(T_{k}^{-1} \xi\right) \leq$ $K$ since $\mathcal{C}$ is a covering and $\left\{T_{k} \mathcal{B}_{A}(0,2 \rho)\right\}_{k}$ is admissible. In fact, $\sum_{k} \Phi\left(T_{k}^{-1} \xi\right)=\sum_{k \in F_{\xi}} \Phi\left(T_{k}^{-1} \xi\right)$ with $F_{\xi}:=\left\{k \in \mathbb{N}: \xi \in T_{k} \mathcal{B}_{A}(0,2 \rho)\right\}$, where the cardinality if $F_{\xi}$ is uniformly bounded in $\xi$. Let $\mu_{j}(\xi)=\varphi_{j}\left(T_{j} \xi\right)$, 
and put $t_{j}:=h\left(\xi_{j}\right)$. We have

$$
\mu_{j}(\xi)=\frac{\Phi(\xi)}{\sum_{k} \Phi\left(T_{k}^{-1} T_{j} \xi\right)}=\frac{\Phi(\xi)}{\sum_{k} \Phi\left(\delta_{t_{k}^{-1}} \delta_{t_{j}} \xi+\delta_{t_{k}^{-1}} \xi_{j}-\delta_{t_{k}^{-1}} \xi_{k}\right)} .
$$

We consider $\partial^{\alpha} \mu_{k}$. For $f(\xi):=\Phi\left(\delta_{t_{k}^{-1}} \delta_{t_{j}} \xi+\delta_{t_{k}^{-1}} \xi_{j}-\delta_{t_{k}^{-1}} \xi_{k}\right)$, the chain rule shows that $\partial^{\eta} f=\sum_{\beta:|\beta|=|\eta|} p_{\beta} \partial^{\beta} \Phi$, where $p_{\beta}$ are monomials of degree $|\eta|$ in the entries of $\delta_{t_{k}^{-1}} \delta_{t_{j}}$. It follows from the estimate (4.2) that

$$
\left|\partial^{\beta} \mu_{j}(\xi)\right| \leq C_{\beta} K^{|\beta|} \chi_{\mathcal{B}_{A}(0,2 \rho)}(\xi), \quad \beta \in \mathbb{N}_{0}^{d},
$$

with $C_{\beta}$ a constant that does not depend on $j$. Thus, we have for any $N \in \mathbb{N}$

$$
\begin{aligned}
\left|\mathcal{F}^{-1} \mu_{k}(x)\right| & \leq C(1+|x|)^{-N}\left|\sum_{|\beta| \leq N} x^{\beta} \mathcal{F}^{-1} \mu_{k}(x)\right| \\
& \leq C(1+|x|)^{-N} \sum_{|\beta| \leq N}\left\|\partial_{\xi}^{\beta} \mu_{k}\right\|_{L_{1}} \\
& \leq C_{N}(1+|x|)^{-N} \leq C_{N}^{\prime}\langle x\rangle_{B}^{-N \alpha_{1}},
\end{aligned}
$$

and (c) follows at once from this estimate.

In a similar fashion,

$$
\psi_{j}(\xi):=\frac{\Phi\left(T_{j}^{-1} \xi\right)}{\sqrt{\sum_{k \in \mathbb{N}} \Phi\left(T_{k}^{-1} \xi\right)^{2}}}
$$

defines a "square root" of the BAPU, where $\left\{\psi_{j}\right\}$ satisfies (c) of Definition 4.8 .

For a BAPU $\left\{\varphi_{k}\right\}_{k \in \mathbb{N}}$ associated with the admissible covering $\left\{T_{k} \mathcal{B}_{A}(0,2 \rho)\right\}_{k \in \mathbb{N}}$ we define

$$
\varphi_{k}^{*}:=\sum_{j \in \tilde{k}} \varphi_{j} \quad \text { and } \quad \varphi_{k}^{K *}:=\sum_{j \in \tilde{k}^{(K)}} \varphi_{j} .
$$

We will use extensively that $\varphi_{k} \varphi_{k}^{*}=\varphi_{k}$.

Now we have a partition of unity associated with fairly general decompositions of $\mathbb{R}^{d}$ and suitable for the definition of T-L spaces. Before we consider the definition of the spaces, we need a class of weight functions.

Definition 4.9. Let $\mathcal{Q}=\left\{Q_{k}\right\}_{k \in \mathbb{N}}$ be an admissible covering. A function $w: \mathbb{R}^{d} \rightarrow(0, \infty)$ is called $\mathcal{Q}$-moderate if there exists $C>0$ such that 
$w(\xi) \leq C w(\zeta)$ for all $\xi, \zeta \in Q_{k}$ and all $k \in \mathbb{N}$. A strictly positive $\mathcal{Q}$ moderate weight (derived from $w$ ) is a sequence $v_{k}=w\left(\xi_{k}\right), k \in \mathbb{N}$, with $\xi_{k} \in Q_{k}$, and $w$ a $\mathcal{Q}$-moderate function.

Example 4.10. For a covering $\mathcal{Q}$ generated by a moderate function $h$, $w=h^{s}, s \in \mathbb{R}$, is a $\mathcal{Q}$-moderate function.

Remark 4.11. For any two coverings $\mathcal{Q}$ and $\mathcal{P}$ of the type considered in Lemma 4.7, it is easy to check that a function $w$ is $\mathcal{Q}$-moderate if and only if it is $\mathcal{P}$-moderate. In the context of such coverings, we will just use the term moderate function/weight.

\section{Triebel-Lizorkin type spaces}

We can now define our new class of T-L type spaces. The spaces will be defined in terms of a moderate function $h$ and a moderate weight $w$. The multiplier results of Section 3 will be called on to show that the spaces are well-defined and independent of the particular partition of unity. For the sake of comparison, we also introduce associated modulation spaces.

Definition 5.1. Let $h$ be a moderate function satisfying $h(\xi) \leq C\langle\xi\rangle_{A}$, and let $\mathcal{Q}$ be an admissible covering generated by $h$ of the type considered in Lemma 4.7. Suppose $\mathcal{T}=\left\{T_{k}\right\}_{k \in \mathbb{N}}, T_{k}=\delta_{h\left(\xi_{k}\right)} \cdot+\xi_{k}$, is the induced family of invertible affine transformations and let $\left\{\varphi_{k}\right\}_{k \in \mathbb{N}}$ be a corresponding BAPU. Suppose $w$ is a moderate function satisfying the growth condition

$$
c_{1}\langle\xi\rangle^{\kappa} \leq w(\xi) \leq c_{2}\langle\xi\rangle^{\gamma}, \quad \xi \in \mathbb{R}^{d},
$$

for some $\kappa, \gamma>0$.

- For $s \in \mathbb{R}, 0<p<\infty$, and $0<q \leq \infty$ we let $F_{p, q}^{s}(h, w)$ denote the set of distributions $f \in \mathcal{S}^{\prime}\left(\mathbb{R}^{d}\right)$ satisfying

$$
\|f\|_{F_{p, q}^{s}(h, w)}:=\left\|\left\{w\left(\xi_{k}\right)^{s} \varphi_{k}(D) f\right\}_{k}\right\|_{L_{p}\left(\ell_{q}\right)}<\infty .
$$

- For $s \in \mathbb{R}$, and $0<p, q \leq \infty$ we let $M_{p, q}^{s}(h, w)$ denote the set of distributions $f \in \mathcal{S}^{\prime}\left(\mathbb{R}^{d}\right)$ satisfying

$$
\|f\|_{M_{p, q}^{s}(h, w)}:=\left\|\left\{w\left(\xi_{k}\right)^{s} \varphi_{k}(D) f\right\}_{k}\right\|_{\ell_{q}\left(L_{p}\right)}<\infty .
$$

It is easy to see that $\|\cdot\|_{F_{p, q}^{s}(h, w)}$ and $\|\cdot\|_{M_{p, q}^{s}(h, w)}$ are quasi-norms (norms if $p, q \geq 1$ ), but it is not so obvious that the spaces are complete. For completeness, the growth conditions imposed on $h$ and $w$ are needed. We have the following result, whose proof can be fund in Appendix A. 
Proposition 5.2. Let $h, w$ be moderate functions satisfying the conditions given in Definition 5.1. For $s \in \mathbb{R}$ and $0<p, q \leq \infty$ :

- $M_{p, q}^{s}(h, w)$ is a quasi-Banach space (Banach space if $1 \leq p, q$ ), and

$$
\mathcal{S}\left(\mathbb{R}^{d}\right) \hookrightarrow M_{p, q}^{s}(h, w) \hookrightarrow \mathcal{S}^{\prime}\left(\mathbb{R}^{d}\right) .
$$

- If $p<\infty$, then $F_{p, q}^{s}(h, w)$ is a quasi-Banach space (Banach space if $1 \leq p, q)$, and

$$
\mathcal{S}\left(\mathbb{R}^{d}\right) \hookrightarrow F_{p, q}^{s}(h, w) \hookrightarrow \mathcal{S}^{\prime}\left(\mathbb{R}^{d}\right) .
$$

Furthermore, if $p<\infty$ and $q<\infty$, then $\mathcal{S}\left(\mathbb{R}^{d}\right)$ is dense in both $M_{p, q}^{s}(h, w)$ and $F_{p, q}^{s}(h, w)$.

Several specific examples of T-L type space will be considered in Section 8. We now focus on the properties of $F_{p, q}^{s}(h, w)$. Notice that since $\mathcal{T}$ is constructed from a moderate function $h$, we have

$$
\|f\|_{F_{p, q}^{s}(h, w)} \asymp\left\|\left\{w\left(\xi_{k}\right)^{s} \varphi_{k}^{* n}(D) f\right\}_{k}\right\|_{L_{p}\left(\ell_{q}\right)}
$$

for any $n \in \mathbb{N}$ with equivalence depending only on $h$ and $n$.

To make sure that $F_{p, q}^{s}(h, w)$ is well defined we need to check that the definition is independent of the particular BAPU.

Proposition 5.3. The space $F_{p, q}^{s}(h, w)$ from Definition 5.1 depends only on $h$ and $w$ up to equivalence of norms.

Proof. Let $\left\{\varphi_{k}\right\}_{k}$ and $\left\{\psi_{k}\right\}_{k}$ be two BAPU's associated with two admissible coverings $\mathcal{Q}$ and $\mathcal{P}$ of the type considered in Lemma 4.7. Suppose $\mathcal{Q}$ and $\mathcal{P}$ are generated by the affine transformations $\left\{T_{k}=\right.$ $\left.\delta_{h\left(\xi_{k}\right)} \cdot+\xi_{k}\right\}_{k}$ and $\left\{U_{k}=\delta_{h\left(\zeta_{j}\right)} \cdot+\zeta_{j}\right\}_{j}$, respectively. Since $\mathcal{Q}$ and $\mathcal{P}$ are equivalent, there exists a function $I: \mathbb{N} \rightarrow \mathbb{N}$ and constants $K, m_{0} \in \mathbb{N}$ such that $\# I^{-1}(j) \leq m_{0}$ for all $j \in \mathbb{N}$ and $\psi_{k}=\psi_{k} \varphi_{I(k)}^{* K}$, for $k \in \mathbb{N}$. This nontrivial claim can be deduced from Lemma 2.9 in [17]. The weight $w$ is moderate so we have $w\left(\zeta_{k}\right) \asymp w\left(\xi_{I(k)}\right)$ uniformly in $k$. Thus, using Theorem 3.5 and (5.2) we get

$$
\begin{aligned}
\left\|\left\{w\left(\zeta_{k}\right)^{s} \psi_{k}(D) f\right\}_{k}\right\|_{L_{p}\left(\ell_{q}\right)} & =\left\|\left\{w\left(\zeta_{k}\right)^{s} \psi_{k}(D) \varphi_{I(k)}^{K *}(D) f\right\}_{k}\right\|_{L_{p}\left(\ell_{q}\right)} \\
& \leq C\left\|\left\{w\left(\xi_{I(k)}\right)^{s} \varphi_{I(k)}^{K *}(D) f\right\}_{k}\right\|_{L_{p}\left(\ell_{q}\right)} \\
& \leq C\left\|\left\{w\left(\xi_{I(k)}\right)^{s} \varphi_{I(k)}^{K *}(D) f\right\}_{k}\right\|_{L_{p}\left(\ell_{q}\right)} \\
& \leq C^{\prime}\left\|\left\{w\left(\xi_{k}\right)^{s} \varphi_{k}(D) f\right\}_{k}\right\|_{L_{p}\left(\ell_{q}\right)},
\end{aligned}
$$

where $C^{\prime}$ depends on the value of $m_{0}$. The converse estimate is obtained by the same arguments, interchanging the role of $\left\{\varphi_{k}\right\}_{k}$ and $\left\{\psi_{k}\right\}_{k}$. 
5.1. Some Properties of $\boldsymbol{F}_{p, q}^{\boldsymbol{s}}(\boldsymbol{h}, \boldsymbol{w})$. Here we present some additional results about the T-L type spaces, which are of some interest in their own right.

5.1.1. An equivalent representation and dual spaces. The following proposition gives a useful equivalent definition of $F_{p, q}^{s}(h, w)$ whenever $1<p, q<\infty$. We will use the representation below to characterize the dual space of $F_{p, q}^{s}(h, w)$ in certain cases.

Proposition 5.4. Suppose $\left\{\varphi_{k}\right\}_{k \in \mathbb{N}}$ is a BAPU corresponding to $\mathcal{T}$, with a sequence of $L_{1}$-majorants $\left\{K_{k}\right\}_{k \in \mathbb{N}}$ as given in Corollary 3.2, satisfying $\sup _{k}\left\|K_{k}\right\|_{L_{1}}<\infty$. Fix $s \in \mathbb{R}$ and $1<p, q<\infty$. For $f \in \mathcal{S}^{\prime}\left(\mathbb{R}^{d}\right)$ let

$$
\mathcal{E}(f)=\left\{\left\{f_{k}\right\}_{k} \subset L_{p}: f=\sum_{k} \varphi_{k}(D) f_{k} \text { in } \mathcal{S}^{\prime}\left(\mathbb{R}^{d}\right)\right\} .
$$

Then$$
F_{p, q}^{s}(h, w)=\left\{f \in \mathcal{S}^{\prime}\left(\mathbb{R}^{d}\right): \exists\left\{f_{k}\right\} \in \mathcal{E}(f) \text { s.t. }\left\|\left\{w\left(\xi_{k}\right)^{s} f_{k}\right\}_{k}\right\|_{L_{p}\left(\ell_{q}\right)}<\infty\right\} .
$$

Moreover,

$$
\|f\|_{F_{p, q}^{s}(h, w)} \asymp \inf _{\left\{f_{k}\right\} \in \mathcal{E}(f)}\left\|\left\{w\left(\xi_{k}\right)^{s} f_{k}\right\}_{k}\right\|_{L_{p}\left(\ell_{q}\right)},
$$

with the convention $\inf _{\emptyset}=\infty$.

Proof. If $f \in F_{p, q}^{s}(h, w)$, then $f$ belongs to the class on the RHS of (5.3), with $f_{k}=\varphi_{k}^{*}(D) f$, and the lower bound in (5.4) holds.

Conversely, suppose $f=\sum_{k} \varphi_{k}(D) f_{k}$ is in the class given by the RHS of (5.3). Then two applications of Corollary 3.2 yields

$$
\begin{aligned}
\|f\|_{F_{p, q}^{s}(h, w)} & =\left\|\left\{w\left(\xi_{k}\right)^{s} \varphi_{k}(D) \sum_{k^{\prime} \in k^{*}} \varphi_{k^{\prime}}(D) f_{k}\right\}_{k}\right\|_{L_{p}\left(\ell_{q}\right)} \\
& \leq c\left\|\left\{w\left(\xi_{k}\right)^{s} f_{k}\right\}_{k}\right\|_{L_{p}\left(\ell_{q}\right)},
\end{aligned}
$$

where $k^{*}=\left\{k^{\prime} \in \mathbb{N}: \operatorname{supp}\left(\varphi_{k}\right) \cap \operatorname{supp}\left(\varphi_{k^{\prime}}\right) \neq \emptyset\right\}$.

Remark 5.5. Notice that if the BAPU satisfies $\left|\partial_{\beta}\left(\varphi_{k}\left(T_{k} \xi\right)\right)\right| \leq$ $C_{\beta} \chi_{Q}(\xi)$ for some fixed compact set $Q$ and for all $|\beta| \leq d+1$, then the majorant condition in Proposition 5.4 is satisfied. In particular, the BAPU defined by (4.3) is admissible.

The characterization of $F_{p, q}^{s}(h, w)$ in Proposition 5.4 can be used to obtain a description of its (topological) dual space. 
Proposition 5.6. If $s \in \mathbb{R}$ and $1<p, q<\infty$, we have

$$
\left(F_{p, q}^{s}(h, w)\right)^{\prime}=F_{p^{\prime}, q^{\prime}}^{-s}(h, w),
$$

where $1=1 / p+1 / p^{\prime}$ and $1=1 / q+1 / q^{\prime}$.

We omit the proof of this result, since it is exactly the same as for the classical T-L spaces (see e.g. [36, $§ 2.11 .2]$ ).

5.1.2. Embedding results. As for classical T-L spaces, we can state some embedding results for the spaces $F_{p, q}^{s}(h, w)$.

Proposition 5.7. Let $0<p<\infty, s \in \mathbb{R}$. Then

$$
F_{p, q_{0}}^{s}(h, w) \hookrightarrow F_{p, q_{1}}^{s}(h, w) \quad \text { for } \quad 0<q_{0} \leq q_{1} \leq \infty,
$$

and

$$
M_{p, q_{0}}^{s}(h, w) \hookrightarrow M_{p, q_{1}}^{s}(h, w) \quad \text { for } \quad 0<q_{0} \leq q_{1} \leq \infty .
$$

(ii) $M_{p, \min \{p, q\}}^{s}(h, w) \hookrightarrow F_{p, q}^{s}(h, w) \hookrightarrow M_{p, \max \{p, q\}}^{s}(h, w)$ for $0<q \leq \infty$.

(iii) Suppose the admissible covering used to define $F_{p, q_{0}}^{s}(h, w)$ is generated by $\mathcal{T}=\left\{T_{k}=\delta_{h\left(\xi_{k}\right)} \cdot+\xi_{k}\right\}_{k}$. Let $0<q_{1} \leq \infty$ and suppose $s_{0}$ is a constant such that $\left\{w\left(\xi_{k}\right)^{-s_{0}}\right\}_{k} \in \ell_{q_{1}}$. Then

$$
M_{p, q_{0}}^{s+s_{0}}(h, w) \hookrightarrow M_{p, q_{1}}^{s}(h, w)
$$

and

$$
F_{p, q_{0}}^{s+s_{0}}(h, w) \hookrightarrow F_{p, q_{1}}^{s}(h, w)
$$

for all $0<q_{0} \leq \infty$.

Proof. The embeddings in (i) are a simple consequence of the monotonicity of the $\ell_{q}$-spaces. The estimate (ii) follows directly from the general estimates (see, e.g., [36, §2.3.2])

$$
\left\|\left\{g_{k}\right\}\right\|_{\ell_{p}\left(L_{p}\right)} \leq\left\|\left\{g_{k}\right\}\right\|_{L_{p}\left(\ell_{q}\right)} \leq\left\|\left\{g_{k}\right\}\right\|_{\ell_{q}\left(L_{p}\right)}, \quad 0<q \leq p<\infty,
$$

and

$$
\left\|\left\{g_{k}\right\}\right\|_{\ell_{q}\left(L_{p}\right)} \leq\left\|\left\{g_{k}\right\}\right\|_{L_{p}\left(\ell_{q}\right)} \leq\left\|\left\{g_{k}\right\}\right\|_{\ell_{p}\left(L_{p}\right)}, \quad 0<p<q \leq \infty .
$$

The final embeddings follow from (i) and the elementary estimate

$$
\left(\sum_{k} w\left(\xi_{k}\right)^{q_{1} s}\left|b_{k}\right|^{q_{1}}\right)^{1 / q_{1}} \leq\left[\sup _{m} w\left(\xi_{m}\right)^{s+s_{0}}\left|b_{m}\right|\right]\left(\sum_{k} w\left(\xi_{k}\right)^{-s_{0} q_{1}}\right)^{1 / q_{1}} .
$$


Remark 5.8. We notice that a set of affine transformations $\mathcal{T}=\left\{T_{k}=\right.$ $\left.\delta_{h\left(\xi_{k}\right)} \cdot+\xi_{k}\right\}_{k}$ generated by a moderate function $h$ using Lemma 4.7 satisfies

$$
0<\inf _{m \neq n}\left|\xi_{m}-\xi_{n}\right|_{A} \leq C \inf _{m \neq n}\left|\xi_{m}-\xi_{n}\right| .
$$

It follows from (5.1) that there exists a constant $\beta:=\beta(\mathcal{T})$ such that $\left\{w\left(\xi_{k}\right)^{-\beta}\right\}_{k} \in \ell_{1}$.

5.1.3. Interpolation. We conclude this section by describing one result about complex interpolation of the family $F_{p, q}^{s}(h, w)$. We note that the interpolation results are consequences of the general theory developed by Triebel in [38].

Proposition 5.9. Suppose $-\infty<s_{0}, s_{1}<\infty, 1<p_{1}, p_{1}, q_{0}, q_{1}<\infty$, $0<\theta<1$. Then

$$
\left[F_{p_{0}, q_{0}}^{s_{0}}(h, w), F_{p_{1}, q_{1}}^{s_{1}}(h, w)\right]_{\theta}=F_{p, q}^{s}(h, w),
$$

where $s=(1-\theta) s_{1}+\theta s_{1}, \quad 1 / p=(1-\theta) / p_{0}+\theta / p_{1}$, and $1 / q=$ $(1-\theta) / q_{0}+\theta / q_{1}$.

Proof. Let $\left\{\varphi_{k}\right\}_{k \in \mathbb{N}}$ be the resolution of the identity given by (4.3). Let $\ell_{q, w}$ denote the weighted sequence space consisting of sequences $\left\{c_{k}\right\}_{k \in \mathbb{N}}$ satisfying $\sum_{k \in \mathbb{N}}\left(w\left(\xi_{k}\right) c_{k}\right)^{q}<\infty$. We define the bounded mapping $S$ : $F_{p, q}^{s}(h, w) \rightarrow L_{p}\left(\ell_{q, w^{s}}\right)$ by

$$
S(f)=\left\{f *\left(\mathcal{F}^{-1} \varphi_{k}\right)\right\}_{k \in \mathbb{N}},
$$

and a corresponding (retract) mapping $R: L_{p}\left(\ell_{q, w^{s}}\right) \rightarrow F_{p, q}^{s}(h, w)$ by

$$
R\left(\left\{f_{k}\right\}\right)=\sum_{k \in \mathbb{N}}\left(\mathcal{F}^{-1} \varphi_{k}^{*}\right) * f_{k}, \quad\left[\text { in } \mathcal{S}^{\prime}\left(\mathbb{R}^{d}\right)\right] .
$$

The boundedness of $R$ follows from Corollary 3.2. It is now straightforward to verify that $R S=I d_{F_{p, q}^{s}(h, w)}$. We now interpolate $S$ and $R$ on the couples $\left\{F_{p_{0}, q_{0}}^{s_{0}}(h, w), F_{p_{1}, q_{1}}^{s_{1}}(h, w)\right\}$ and $\left\{L_{p_{0}}\left(\ell_{q_{0}, w^{s_{0}}}\right), L_{p_{1}}\left(\ell_{q_{1}, w^{s_{1}}}\right)\right\}$. We have,

$$
\left[\ell_{q_{0}, w^{s_{0}}}, \ell_{q_{1}, w^{s_{1}}}\right]_{\theta}=\ell_{q, w^{s}}
$$

which can be deduced from [38, Theorem 1.18.1]. Using this result we apply [38, Theorem 1.18.4] to obtain

$$
\left[L_{p_{0}}\left(\ell_{q_{0}, w^{s_{0}}}\right), L_{p_{1}}\left(\ell_{q_{1}, w^{s_{1}}}\right)\right]_{\theta}=L_{p}\left(\ell_{q, w^{s}}\right) .
$$

The boundedness of $R$ and $S$ shows that $\left[F_{p_{0}, q_{0}}^{s_{0}}(h, w), F_{p_{1}, q_{1}}^{s_{1}}(h, w)\right]_{\theta}$ is isomorphic to the closed subspace $F_{p, q}^{s}(h, w)$ of $L_{p}\left(\ell_{q, w^{s}}\right)$. 


\section{Characterization by tight frames}

Here we present an easy construction of a tight frame for $L_{2}\left(\mathbb{R}^{d}\right)$ that gives a stable discrete representation and characterization of the T-L type spaces. The particular method used below to construct tight frames was introduced by the authors in [6].

It is useful to have discrete expansions of the T-L type spaces for several reasons. We obtain an explicit method to estimate the T-L type norm of a function $f$ simply by calculating the frame coefficients of $f$. The characterization also provides a one to one correspondence between smoothness of a function and sparseness of its frame coefficients, something that is very useful for approximation purposes. Another important application of discrete decompositions is to simplify the analysis of operators acting on it. For example, we can discretize the pseudo-differential operators studied in Section 7 below using the tight frame.

Let us now define the tight frame. Consider the modified BAPU $\left\{\psi_{k}\right\}_{k \in \mathbb{N}}$ given by (4.6) associated with the admissible covering $\mathcal{C}=\left\{T_{k} \mathcal{B}_{A}(0, \rho)\right\}_{k \in \mathbb{N}}$ generated by $\left\{T_{k}=\delta_{h\left(\xi_{k}\right)}++\xi_{k}\right\}_{k \in \mathbb{N}}$. Suppose $K_{a}$ is a cube in $\mathbb{R}^{d}$ (aligned with the coordinate axes) with side-length $2 a$ satisfying $\mathcal{B}_{A}(0,2 \rho) \subseteq K_{a}$. Put $t_{k}=h\left(\xi_{k}\right)$. Then we define

$$
e_{k, n}(\xi):=(2 a)^{-\frac{d}{2}} t_{k}^{-\nu / 2} \chi_{K_{a}}\left(T_{k}^{-1} \xi\right) e^{i \frac{\pi}{a} n \cdot T_{k}^{-1} \xi}, \quad n \in \mathbb{Z}^{d}, k \in \mathbb{N} .
$$

and

$$
\hat{\eta}_{k, n}:=\psi_{k} e_{k, n} \quad n \in \mathbb{Z}^{d}, k \in \mathbb{N} .
$$

It is straightforward to verify that $\left\{\eta_{k, n}\right\}_{k, n}$ is a tight frame for $L_{2}\left(\mathbb{R}^{d}\right)$. We just have to notice that $\left\{e_{k, n}\right\}_{n \in \mathbb{Z}^{d}}$ is an orthonormal basis for $L_{2}\left(T_{k}\left(K_{a}\right)\right)$ and $\operatorname{supp}\left(\psi_{k}\right) \subset T_{k}\left(K_{a}\right)$ which yields

$$
\sum_{n \in \mathbb{Z}^{d}}\left|\left\langle f, \eta_{k, n}\right\rangle\right|^{2}=\sum_{n \in \mathbb{Z}^{d}}\left|\left\langle\psi_{k} \hat{f}, e_{k, n}\right\rangle\right|^{2}=\left\|\psi_{k} \hat{f}\right\|_{L_{2}}^{2} .
$$

Moreover, since $\left\{\psi_{k}^{2}\right\}_{k \in \mathbb{N}}$ is a partition of unity,

$$
\sum_{n \in \mathbb{Z}^{d}, k \in \mathbb{N}}\left|\left\langle f, \eta_{k, n}\right\rangle\right|^{2}=\sum_{k \in \mathbb{N}}\left\|\psi_{k} \hat{f}\right\|_{L_{2}}^{2}=\int_{\mathbb{R}^{d}} \sum_{k \in \mathbb{N}} \psi_{k}^{2}(\xi)|\hat{f}(\xi)|^{2} d \xi=\|f\|_{L_{2}}^{2} .
$$

We can also obtain an explicit representation of $\eta_{k, n}$ in direct space. Put $\hat{\mu}_{k}(\xi):=\psi_{k}\left(T_{k} \xi\right)$. Then

$$
\eta_{k, n}(x)=(2 a)^{-\frac{d}{2}} t_{k}^{\nu / 2} \mu_{k}\left(\frac{\pi}{a} n+\delta_{t_{k}}^{\top} x\right) e^{i x \cdot \xi_{k}} .
$$


By similar estimates as for the inequality (4.5), it can be verified that

$$
\left|\mu_{k}(x)\right| \leq C_{N}\langle x\rangle_{B}^{-N},
$$

for any $N \in \mathbb{N}$, with $C_{N}$ independent of $x \in \mathbb{R}^{d}$ and $k \in \mathbb{N}$.

We notice that $\eta_{k, n}$ is obtained by translating, dilating, and modulating a unit-scale element $\mu_{k}$. More precisely, we translate by $\frac{\pi}{a} n$, modulate by $e^{i x \cdot \xi_{k}}$, and then dilate by $\delta_{t_{k}}^{\top}$. So, in some sense, $\eta_{k, n}$ is a mix between a Gabor and a wavelet system. The dilation and translation structure is a feature also found in a generic wavelet system $\left\{|M|^{1 / 2} \psi\left(M^{j} x-k\right)\right\}_{j, k}$ associated with a dilation matrix $M$. Modulation is an integral part of generic Gabor systems, $\left\{g(x-n a) e^{i b m \cdot x}\right\}_{m, n}$ and in (6.2) the features are combined with dilation to form "mixed" atoms.

Our next goal is to show that the frame expansion gives an atomic decomposition of the spaces $F_{p, q}^{s}(h, w)$. By an atomic decomposition, we mean that the canonical coefficient operator is bounded on $F_{p, q}^{s}(h, w)$ into a suitable coefficient space on which there is a bounded reconstruction operator. To introduce a suitable sequence space, we first define the point sets

$$
Q(k, n)=\left\{y \in \mathbb{R}^{d}: \delta_{t_{k}}^{\top} y+\frac{\pi}{a} n \in \mathcal{B}_{B}(0,1)\right\} .
$$

It is easy to verify there exists $L<\infty$ so that uniformly in $x$ and $k$, $\sum_{n} \chi_{Q(k, n)}(x) \leq L$.

We can now prove that the canonical coefficient operator is bounded on $F_{p, q}^{s}(h, w)$.

Lemma 6.1. Let $\left\{T_{k}=\delta_{h\left(\xi_{k}\right)} \cdot+\xi_{k}\right\}_{k \in \mathbb{N}}$ be a family of invertible affine transformations based on a moderate function $h$. Suppose $s \in \mathbb{R}$, $0<p<\infty$, and $0<q \leq \infty$. Then

$$
\left\|S_{q}^{s}(f)\right\|_{L_{p}} \leq C\|f\|_{F_{p, q}^{s}(h, w)}, \quad f \in F_{p, q}^{s}(h, w),
$$

where

$$
S_{q}^{s}(f):=\left(\sum_{k} \sum_{n \in \mathbb{Z}^{d}}\left(w\left(\xi_{k}\right)^{s}\left|\left\langle f, \eta_{k, n}\right\rangle_{A}\right|\left|T_{k}\right|^{1 / 2} \chi_{Q(k, n)}\right)^{q}\right)^{1 / p},
$$

with $Q(k, n)$ given in (6.3).

Proof. Take $f \in F_{p, q}^{s}(h, w)$. Notice that (see Equation (6.1))

$$
\left|T_{k}\right|^{1 / 2}\left|\left\langle f, \eta_{k, n}\right\rangle\right|=(2 a)^{-d / 2}\left|\left(\check{\psi}_{k} * f\right)\left(\frac{\pi}{a} \delta_{t_{k}}^{-\top} n\right)\right|,
$$


where we let $t_{k}=h\left(\xi_{k}\right)$. Moreover, if $Q(k, n) \cap Q\left(k, n^{\prime}\right) \neq \emptyset$ and $u \in Q(k, n), v \in Q\left(k, n^{\prime}\right)$ then $|u-v|_{B} \leq K t_{k}^{-1}$ for some uniform constant $K$. Hence,

$$
\begin{aligned}
& \sum_{n \in \mathbb{Z}^{d}}\left(\left|\left\langle f, \eta_{k, n}\right\rangle\right|\left|T_{k}\right|^{1 / 2} \chi_{Q(k, n)}(x)\right)^{q} \\
& \leq C \sum_{n \in \mathbb{Z}^{d}}\left(\sup _{y \in Q(k, n)}\left|\left(\psi_{k}(D) f\right)(y)\right| \chi_{Q(k, n)}(x)\right)^{q} \\
& \leq C^{\prime} \sup _{z \in \mathcal{B}_{B}\left(0, K t_{k}^{-1}\right)}\left(\left\langle\delta_{t_{k}}^{\top} z\right\rangle_{B}^{-\nu / r}\left|\left(\psi_{k}(D) f\right)(x-z)\right|\right)^{q} \cdot\left\langle\delta_{t_{k}}^{\top} z\right\rangle_{B}^{\nu q / r} \\
& \leq C^{\prime \prime}\left(\sup _{z \in \mathbb{R}^{d}}\left\langle\delta_{t_{k}}^{\top} z\right\rangle_{B}^{-\nu / r}\left|\left(\psi_{k}(D) f\right)(x-z)\right|\right)^{q} .
\end{aligned}
$$

Recall that $\operatorname{supp}\left(\psi_{k}\right) \subset T_{k} \mathcal{C}$, so by Corollary 3.4, Theorem 3.5, and the estimate above,

$$
\begin{aligned}
\left\|S_{q}^{s}(f)\right\|_{L_{p}} & \leq C\left\|\left\{w\left(\xi_{k}\right)^{s} \psi_{k}(D) f\right\}_{k}\right\|_{L_{p}\left(\ell_{q}\right)} \\
& =C\left\|\left\{w\left(\xi_{k}\right)^{s} \psi_{k}(D) \varphi_{k}^{*}(D) f\right\}_{k}\right\|_{L_{p}\left(\ell_{q}\right)} \\
& \leq C^{\prime}\left\|\left\{w\left(\xi_{k}\right)^{s} \varphi_{k}^{*}(D) f\right\}_{k}\right\|_{L_{p}\left(\ell_{q}\right)} \\
& \leq C^{\prime \prime}\|f\|_{F_{p, q}^{s}(h, w)}
\end{aligned}
$$

Remark 6.2. For $1<p, q<\infty$, the converse inequality $\|f\|_{F_{p, q}^{s}(h, w)} \leq$ $C\left\|S_{q}^{s}(f)\right\|_{L_{p}}$ can be obtained by a standard duality argument,

$$
\begin{aligned}
\|f\|_{F_{p, q}^{s}(h, w)} & =\sup _{g \in \mathcal{S}\left(\mathbb{R}^{d}\right):\|g\|_{F_{p^{\prime}, q^{\prime}}^{-s}(h, w)} \leq 1}|\langle f, g\rangle| \\
& \leq c^{\prime}\left\|S_{q}^{S}(f)\right\|_{L_{p}}\left\|S_{q^{\prime}}^{-s}(g)\right\|_{L_{p^{\prime}}}, \quad f \in \mathcal{S}\left(\mathbb{R}^{d}\right),
\end{aligned}
$$

with $1 / p+1 / p^{\prime}=1$ and $1 / q+1 / q^{\prime}=1$, using the characterization of the dual space from Proposition 5.6.

Inspired by Lemma 6.1 , we define the sequence space $f_{p, q}^{s}:=f_{p, q}^{s}(h, w)$ for $s \in \mathbb{R}, 0<p<\infty$, and $0<q \leq \infty$, as the set of sequences $\left\{s_{k, n}\right\}_{k \in \mathbb{N}, n \in \mathbb{Z}^{d}} \subset \mathbb{C}$ satisfying

$$
\left\|\left\{s_{k, n}\right\}\right\|_{f_{p, q}^{s}}:=\left\|\left\{w\left(\xi_{k}\right)^{s}\left|T_{k}\right|^{1 / 2}\left(\sum_{n \in \mathbb{Z}^{d}}\left|s_{k, n}\right|^{q} \chi_{Q(k, n)}\right)^{1 / q}\right\}_{k}\right\|_{L_{p}\left(\ell_{q}\right)}<\infty .
$$


Lemma 6.1 provides us with a bounded coefficient operator $C: F_{p, q}^{s}(h, w) \rightarrow$ $f_{p, q}^{s}$ given by

$$
C f=\left\{\left\langle f, \eta_{k, n}\right\rangle\right\}_{k \in \mathbb{N}, n \in \mathbb{Z}^{d}} .
$$

Moreover, the fact that $\left\{\eta_{k, n}\right\}$ is a tight frame shows that the most reasonable definition of a reconstruction operator is given by

$$
R:\left\{s_{k, n}\right\}_{k, n} \rightarrow \sum_{k, n} s_{k, n} \eta_{k, n} .
$$

Using Lemma A.1 we now verify that $R: f_{p, q}^{s} \rightarrow F_{p, q}^{s}(h, w)$ is also a bounded operator.

Lemma 6.3. Suppose $s \in \mathbb{R}, 0<p<\infty$, and $0<q \leq \infty$. Then for any finite sequence $\left\{s_{k, n}\right\}_{k, n}$, we have

$$
\left\|\sum_{k, n} s_{k, n} \eta_{k, n}\right\|_{F_{p, q}^{s}(h, w)} \leq C\left\|\left\{s_{k, n}\right\}\right\|_{f_{p, q}^{s}} .
$$

Proof. Let $\left\{\varphi_{k}\right\}_{k \in \mathbb{N}}$ be a BAPU associated with $F_{p, q}^{s}(h, w)$. By (6.1) and Theorem 3.5 we get

$$
\begin{aligned}
\left\|\sum_{k, n} s_{k, n} \eta_{k, n}\right\|_{F_{p, q}^{s}} & =\left\|\left\{w\left(\xi_{k}\right)^{s} \varphi_{k}(D)\left(\sum_{\ell, n} s_{\ell, n} \eta_{\ell, n}\right)\right\}_{k}\right\|_{L_{p}\left(\ell_{q}\right)} \\
& \leq C\left\|\left\{w\left(\xi_{k}\right)^{s} \sum_{\ell \in N(k)} \sum_{n} s_{\ell, n} \eta_{\ell, n}\right\}_{k}\right\|_{L_{p}\left(\ell_{q}\right)},
\end{aligned}
$$

where $N(k)=\left\{\ell \in \mathbb{N}: \operatorname{supp}\left(\varphi_{k}\right) \cap \operatorname{supp}\left(\psi_{\ell}\right) \neq \emptyset\right\}$. Since $\# N(k)$ is uniformly bounded by Theorem 4.3 , and $w$ is a moderate weight, we obtain

$$
\begin{aligned}
& \left\|\left\{w\left(\xi_{k}\right)^{s} \sum_{\ell \in N(k)} \sum_{n} s_{\ell, n} \eta_{\ell, n}\right\}_{k}\right\|_{L_{p}\left(\ell_{q}\right)} \\
& \quad \leq C\left\|\left(\sum_{\ell}\left(w\left(\xi_{\ell}\right)^{s} \sum_{n}\left|s_{\ell, n}\right|\left|\eta_{\ell, n}\right|\right)^{q}\right)^{1 / q}\right\|_{L_{p}} .
\end{aligned}
$$

Fix $0<r<\min (1, p, q)$. Then Lemma A.1 and the Fefferman-Stein maximal inequality (3.1) yields

$$
\left\|\left\{w\left(\xi_{k}\right)^{s} \sum_{n}\left|s_{k, n}\right|\left|\eta_{k, n}\right|\right\}_{k}\right\|_{L_{p}\left(\ell_{q}\right)}
$$




$$
\begin{aligned}
& \leq C\left\|\left\{w\left(\xi_{k}\right)^{s}\left|T_{k}\right|^{1 / 2} M_{r}^{B}\left(\sum_{n}\left|s_{k, n}\right| \chi_{Q(k, n)}\right)\right\}_{k}\right\|_{L_{p}\left(\ell_{q}\right)} \\
& \leq C^{\prime}\left\|\left\{w\left(\xi_{k}\right)^{s}\left|T_{k}\right|^{1 / 2} \sum_{n}\left|s_{k, n}\right| \chi_{Q(k, n)}\right\}_{k}\right\|_{L_{p}\left(\ell_{q}\right)} .
\end{aligned}
$$

The result now follows since the sum over $n$ is locally finite with a uniform bound on the number of non-zero terms, which implies that

$$
\left(\sum_{n}\left|s_{k, n}\right| \chi_{Q(k, n)}\right)^{q} \asymp \sum_{n}\left|s_{k, n}\right|^{q} \chi_{Q(k, n)},
$$

uniformly in $k$.

Combining Lemma 6.1 and Lemma 6.3 we obtain that $\left\{\eta_{k, n}\right\}$ forms an atomic decomposition for the spaces $F_{p, q}^{s}(h, w)$.

Theorem 6.4. Given $s \in \mathbb{R}, 0<p, q<\infty$. Then the coefficient operator $C$ given by (6.4) and reconstruction operator $R$ given by (6.5) are both bounded and makes $F_{p, q}^{s}(h, w)$ a retract of $f_{p, q}^{s}$, i.e., $R C=\operatorname{Id}_{F_{p, q}^{s}(h, w)}$. In particular, if $1 \leq p, q<\infty,\left\{\eta_{k, n}\right\}$ is an atomic decomposition of the Banach space $F_{p, q}^{s}(h, w)$.

The retract result in Theorem 6.4 can be illustrated by the following commuting diagram.

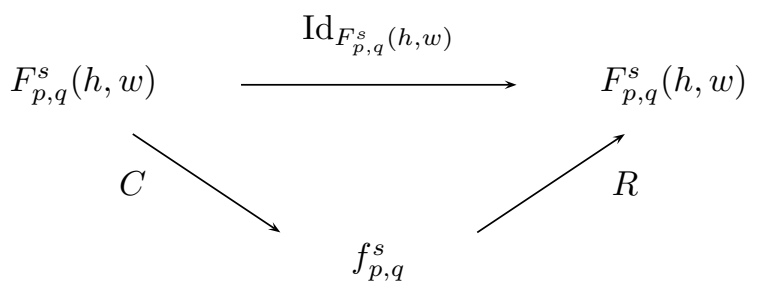

Remark 6.5. Lemma 6.1 and Lemma 6.3 provides the following norm characterization

$$
\|f\|_{F_{p, q}^{s}(h, w)} \asymp\left\|\left\{\left\langle f, \eta_{k, n}\right\rangle\right\}_{k, n}\right\|_{f_{p, q}^{s},}, \quad f \in F_{p, q}^{s}(h, w),
$$

for $s \in \mathbb{R}, 0<p<\infty$, and $0<q \leq \infty$.

\section{Pseudo-differential operators on $F_{p, q}^{s}(h, w)$}

This section contains our main application of the T-L type spaces defined in Section 5. We study boundedness properties of pseudo-differential operators on the T-L type spaces. For convenience, we assume that the 
matrix $A$ has been scaled such that $\alpha_{1}=1 \leq \alpha_{2}$. Notice that in particular, $|\cdot|_{A} \leq|\cdot|$. if

We say that a smooth function $w$ on $\mathbb{R}^{d}$ is an admissible weight function

- $1 \leq w(\xi) \leq C\langle\xi\rangle$ for $\xi \in \mathbb{R}^{d}$

- $\left|\partial^{\beta} w(\xi)\right| \leq C_{\beta} w(\xi)^{1-|\beta|}$ for $\beta \in \mathbb{N}_{0}^{d}$.

Example 7.1. The weight $w(\xi):=\langle\xi\rangle_{A}$ is admissible (since $\alpha_{1}=1$, see part (e) of Lemma 2.3).

It was noticed in [27] that for an admissible weight $w$, there exist $K, R>0$ such that

$$
R^{-1} \leq \frac{w(\zeta)}{w(\xi)} \leq R \quad \text { for } \quad|\xi-\zeta| \leq K w(\xi) .
$$

In particular, since $|\xi-\zeta|_{A} \leq|\xi-\zeta|,(7.1)$ implies that $w$ is a moderate function.

Definition 7.2. For $b \in \mathbb{R}$ and $0<\rho \leq 1$ we define the Hörmander class $S_{w ; \rho}^{b}\left(\mathbb{R}^{d} \times \mathbb{R}^{d}\right)$ as the family of functions $\sigma \in C^{\infty}\left(\mathbb{R}^{d} \times \mathbb{R}^{d}\right)$ satisfying

$$
|\sigma|_{N, M}^{(b)}:=\max _{|\alpha| \leq N,|\beta| \leq M} \sup _{x, \xi \in \mathbb{R}^{d}} w(\xi)^{\rho|\alpha|-b}\left|\partial_{\xi}^{\alpha} \partial_{x}^{\beta} \sigma(x, \xi)\right|<\infty,
$$

for $M, N \in \mathbb{N}$.

The class $S_{w ; \rho}^{b}\left(\mathbb{R}^{d} \times \mathbb{R}^{d}\right)$ has been considered earlier by several authors, see e.g. [27]. We remark that the class is completely independent of the spaces defined in Section 5, and our claim is that the T-L type space can be adapted to study the boundedness properties of the pseudo-differential operators induced by $S_{w ; \rho}^{b}\left(\mathbb{R}^{d} \times \mathbb{R}^{d}\right)$.

Remark 7.3. For the particular choice $w=\langle\cdot\rangle, S_{\langle\cdot\rangle ; \rho}^{b}\left(\mathbb{R}^{d} \times \mathbb{R}^{d}\right)$ is the classical Hörmander class $S_{\rho, 0}^{b}\left(\mathbb{R}^{d} \times \mathbb{R}^{d}\right)$. For a general weight $w$ and $b \leq 0$, we notice that $S_{\langle\cdot\rangle ; \rho}^{b}\left(\mathbb{R}^{d} \times \mathbb{R}^{d}\right) \subset S_{w ; \rho}^{b}\left(\mathbb{R}^{d} \times \mathbb{R}^{d}\right)$ since $w \leq\langle\cdot\rangle$. Therefore, $S_{w ; \rho}^{b}\left(\mathbb{R}^{d} \times \mathbb{R}^{d}\right)$ often contains symbols not included in the standard class.

Given $\sigma \in S_{w ; \rho}^{b}\left(\mathbb{R}^{d} \times \mathbb{R}^{d}\right)$, we define the operator $\sigma(x, D)$ in terms of the symbol $\sigma(x, \xi)$ by

$$
\sigma(x, D) f(x):=\frac{1}{(2 \pi)^{d / 2}} \int_{\mathbb{R}^{d}} \sigma(x, \xi) \hat{f}(\xi) e^{i x \cdot \xi} d \xi, \quad f \in \mathcal{S}\left(\mathbb{R}^{d}\right) .
$$

The family of all operators induced by $S_{w ; \rho}^{b}\left(\mathbb{R}^{d} \times \mathbb{R}^{d}\right)$ is denoted Op $S_{w ; \rho}^{b}$. For $\sigma \in S_{w ; \rho}^{b}\left(\mathbb{R}^{d} \times \mathbb{R}^{d}\right)$, we notice that $\sigma(x, D): \mathcal{S}\left(\mathbb{R}^{d}\right) \rightarrow \mathcal{S}\left(\mathbb{R}^{d}\right)$ which 
follows by standard arguments since $w(\xi) \leq C\langle x\rangle$ for all $\xi \in \mathbb{R}^{d}$. Our goal is now to find sufficient conditions on a moderate weight $h$ to ensure that

$$
\sigma(x, D): F_{p, q}^{s}(h, w) \rightarrow F_{p, q}^{s-b}(h, w) .
$$

Let us state the main result. The proof will be given at the end of the section.

Theorem 7.4. Given $\sigma \in S_{w ; \rho}^{b}\left(\mathbb{R}^{d} \times \mathbb{R}^{d}\right), 0<\rho \leq 1, b \in \mathbb{R}$. Suppose the moderate weight $h$ satisfies

$$
C_{1}\langle\xi\rangle_{A}^{\kappa} \leq h(\xi) \leq C_{2} w(\xi)^{\rho / \alpha_{2}} \quad \forall \xi \in \mathbb{R}^{d},
$$

for some $\kappa>0$. Then $\sigma(x, D)$ extends to a bounded operator

$$
\sigma(x, D): F_{p, q}^{s+b}(h, w) \rightarrow F_{p, q}^{s}(h, w) .
$$

for any $s \in \mathbb{R}$, and $p, q \in[1, \infty)$.

Remark 7.5. We need to be concerned that spaces $F_{p, q}^{s}(h, w)$ used in Theorem 7.4 are well-defined in order for the statement of the theorem to make sense. Let us elaborate on this point. We have $h(\xi) \leq C_{2} w(\xi)$ by $(7.3)$ since $\rho / \alpha_{2} \leq 1$ and $w \geq 1$. Hence, (7.1) shows that there are constants $K, R>0$ such that

$$
R^{-1} \leq \frac{w(\zeta)}{w(\xi)} \leq R \quad \text { for } \quad|\xi-\zeta|_{A} \leq \frac{K}{C_{2}} h(\xi), \quad \xi, \zeta \in \mathbb{R}^{d} .
$$

That is, $w$ is a $\mathcal{Q}$-moderate weight for any covering $\mathcal{Q}$ generated by $h$ if we choose the moderation constant $\rho$ for $h$ sufficiently small. For such a choice, the spaces $F_{p, q}^{s}(h, w)$ are well-defined.

We follow the same strategy as in $[37, \S 6.2 .2]$ for the proof of Theorem 7.4 (see also [29]). The plan of attack is to reduce the proof to the case $b=0$ and $s$ large. We begin this process by studying the Fourier multiplier case. We have the following result.

Proposition 7.6. Let $w$ be a moderate weight, and suppose $\sigma$ is a smooth function for which there exists $b \in \mathbb{R}$ and $0<\rho \leq 1$ such that

$$
\left|\partial^{\beta} \sigma(\xi)\right| \leq C_{\beta} w(\xi)^{b-|\beta| \rho}
$$

for all $\beta \in \mathbb{N}^{d}$. Then for any moderate weight $h$ satisfying (7.3), $\sigma(D)$ extends to a bounded operator

$$
\sigma(D): F_{p, q}^{s}(h, w) \rightarrow F_{p, q}^{s-b}(h, w)
$$


for any $s \in \mathbb{R}$, and $p, q \in[1, \infty)$.

Proof. For $T_{k}=\delta_{h\left(\xi_{k}\right)}++\xi_{k} \in \mathcal{T}$ let $\sigma_{k}(\xi)=w\left(\xi_{k}\right)^{-b} \sigma(\xi) \varphi_{k}^{*}(\xi)$, and $\mu_{k}(\xi)=\sigma_{k}\left(T_{k} \xi\right)$. Notice that for any $\alpha \in \mathbb{N}^{d}$ we have

$$
\partial^{\alpha}\left(\sigma\left(T_{k} \xi\right)\right)=\sum_{|\beta|=|\alpha|} b_{\beta} \partial^{\beta} \sigma\left(T_{k} \xi\right)
$$

where $b_{\beta}$ are monomials of degree $|\beta|$ in the entries of $\delta_{h\left(\xi_{k}\right)}$. Since $h(\xi) \geq \varepsilon_{0}>0$, each entry is bounded by $h\left(\xi_{k}\right)^{\alpha_{2}}$ (up to a constant). Thus

$$
\left|\partial^{\alpha}\left(\sigma\left(T_{k} \xi\right)\right)\right| \leq C_{\alpha} h\left(\xi_{k}\right)^{\alpha_{2}|\alpha|} \sum_{|\beta|=|\alpha|}\left|\partial^{\beta} \sigma\left(T_{k} \xi\right)\right| \leq C_{\alpha}^{\prime} h\left(\xi_{k}\right)^{\alpha_{2}|\alpha|} w\left(T_{k} \xi\right)^{b-\rho|\alpha|},
$$

where we have used the bound (7.4). Now, using (7.3) and that $w$ is a moderate weight, it is straight forward to show that

$$
\left|\partial^{\beta} \mu_{k}(\xi)\right| \leq C_{\beta} \chi_{Q}(\xi),
$$

for $Q$ a fixed compact set and for some constant $C_{\beta}$ independent of $k$. Let $r$ be a constant satisfying $r>\frac{\nu}{2}+\frac{\nu}{\min (p, q)}$. Then by Theorem 3.5 we have

$$
\begin{aligned}
\|\sigma(D) f\|_{F_{p, q}^{s}(h, w)} & =\left\|\left\{\sigma_{k}(D)\left(w\left(\xi_{k}\right)^{s+b} \varphi_{k}(D) f\right)\right\}_{k}\right\|_{L_{p}\left(\ell_{q}\right)} \\
& \leq C \sup _{k}\left\|\sigma_{k}\left(T_{k} \cdot\right)\right\|_{H_{2}^{r}} \cdot\|f\|_{F_{p, q}^{s+b}(h, w)} \\
& \leq C^{\prime}\|f\|_{F_{p, q}^{s+b}(h, w)}
\end{aligned}
$$

for all $f \in F_{p, q}^{s+b}(h, w)$.

The next example will be needed for the proof of Theorem 7.4.

Example 7.7. It is easy to verify that $w(\xi)^{b} \in S_{w ; 1}^{b}$ for $b \in \mathbb{R}$. Let $\mathcal{J}^{s}=w^{s}(D)$. With $h$ as in Proposition 7.6, $\mathcal{J}^{b}: F_{p, q}^{s+b}(h, w) \rightarrow F_{p, q}^{s}(h, w)$ and we have

$$
\left\|\mathcal{J}^{b} f\right\|_{F_{p, q}^{s}(h, w)} \asymp\|f\|_{F_{p, q}^{s+b}(h, w)}, \quad \forall f \in F_{p, q}^{s+b}(h, w),
$$

for any $s \in \mathbb{R}$, and $p, q \in[1, \infty)$.

We can use Example 7.7 in conjunction with Theorem 7.8 below to reduce the proof of Theorem 7.4 to the case $b=0$ and $s$ large. See [1] or [27, Chapter 5] for a proof of Theorem 7.8.

Theorem 7.8. Let $a$ and $b$ be symbols belonging to $S_{w ; \rho}^{m_{1}}$ and $S_{w ; \rho}^{m_{2}}$, respectively, for some $m_{1}, m_{2} \in \mathbb{R}$ and $\rho \in(0,1]$. Then there is a symbol 
$\sigma \in S_{w ; \rho}^{m_{1}+m_{2}}$ so that $\sigma(x, D)=a(x, D) b(x, D)$. Moreover,

$$
\sigma-\sum_{|\alpha|<N} \frac{1}{i^{|\alpha|} \alpha !} \partial_{\xi}^{\alpha} a \cdot \partial_{x}^{\alpha} b \in S_{w ; \rho}^{m_{1}+m_{2}-\rho N}
$$

for all $N \in \mathbb{N}$.

We can now state and prove the final technical lemma before we turn to the proof of Theorem 7.4. We let $\check{f}$ denote the inverse Fourier transform of $f$.

Lemma 7.9. Given $\sigma \in S_{w ; \rho}^{0}, 0<\rho$. Suppose the moderate weight $h$ satisfies (7.3), and let $\left\{\varphi_{k}\right\}$ be the BAPU defined in (4.3) associated with $h$ generated by the affine transformations $\left\{\delta_{h\left(\xi_{k}\right)} \cdot+\xi_{k}\right\}_{k}$. Then

(a) For $|\gamma|,|\alpha| \leq K$ and $J \in \mathbb{N}$ there exists a constant $C:=C(K, J)$ such that

$$
\begin{gathered}
F(x):=\sup _{z \in \mathbb{R}^{d}}\left|\left(\partial_{x}^{\gamma} \sigma(z, \cdot) \partial_{\xi}^{\alpha} \varphi_{k}\right)^{\vee}(x)\right| \leq C|\sigma|_{M, K}^{(0)}\left|\delta_{h\left(\xi_{k}\right)}^{\top}\right|\left\langle\delta_{h\left(\xi_{k}\right)}^{\top} x\right\rangle_{B}^{-J}, \\
x \in \mathbb{R}^{d}, k \in \mathbb{N},
\end{gathered}
$$

for any $M>J$.

(b) For $|\gamma|,|\alpha| \leq K$ and $m \geq 0$ there exists a constant $C^{\prime}:=C^{\prime}(K, m)$, such that

$$
I:=\int_{\mathbb{R}^{d}} \sup _{z \in \mathbb{R}^{d}}\left|\left(\partial_{x}^{\gamma} \sigma(z, \cdot) \partial_{\xi}^{\alpha} \varphi_{k}\right)^{\vee}(x)\right|\langle x\rangle_{B}^{m} d x \leq C^{\prime}|\sigma|_{M, K}^{(0)}, \quad k \in \mathbb{N},
$$

for any $M \in \mathbb{N}$ satisfying $M>m+\nu$.

Proof. First we prove (a). For notational convenience, let $\sigma^{\gamma}(x, \xi):=$ $\partial_{x}^{\gamma} \sigma(x, \xi)$. We have the equality

$$
F(x)=(2 \pi)^{-d / 2} \sup _{z \in \mathbb{R}^{d}}\left|\int_{\mathbb{R}^{d}} e^{i x \cdot \xi} \sigma^{\gamma}(z, \xi) \partial_{\xi}^{\alpha} \varphi_{k}(\xi) d \xi\right| .
$$

Let $T_{k}=\delta_{h\left(\xi_{k}\right)} \cdot+\xi_{k}$. Then a substitution yields

$$
F(x)=(2 \pi)^{-d / 2}\left|\delta_{h\left(\xi_{k}\right)}^{\top}\right| \sup _{z \in \mathbb{R}^{d}}\left|\int_{\mathbb{R}^{d}} e^{i \delta_{h\left(\xi_{k}\right)}^{\top} x \cdot \xi} \sigma^{\gamma}\left(z, T_{k} \xi\right) \partial_{\xi}^{\alpha} \varphi_{k}\left(T_{k} \xi\right) d \xi\right|
$$

Fix $M>J$. Notice that $\langle x\rangle_{B} \leq\langle x\rangle$, so we have the standard estimate $\langle x\rangle_{B}^{M}|\hat{g}(x)| \leq C_{M} \sum_{|\beta| \leq K}\left\|\partial^{\beta} g\right\|_{L_{1}}$, for some finite constant $C_{M}$. We apply 
this estimate to (7.7) to obtain

$$
\begin{aligned}
& F\left(\delta_{h\left(\xi_{k}\right)}^{-\top} x\right) \\
& \quad \leq C_{M}(2 \pi)^{-d / 2}\left|\delta_{h\left(\xi_{k}\right)}^{\top}\right| \sup _{z \in \mathbb{R}^{d}} \sum_{|\beta| \leq M} \int_{\mathbb{R}^{d}}\left|\partial_{\xi}^{\beta}\left[\sigma^{\gamma}\left(z, T_{k} \xi\right) \partial_{\xi}^{\alpha} \varphi_{k}\left(T_{k} \xi\right)\right]\right| d \xi\langle x\rangle_{B}^{-J},
\end{aligned}
$$

which by Leibniz's rule provides the bound

$$
\begin{aligned}
& F\left(\delta_{h\left(\xi_{k}\right)}^{-\top} x\right) \\
& \quad \leq C^{\prime}\left|\delta_{h\left(\xi_{k}\right)}^{\top}\right| \sum_{\substack{|\beta| \leq M \\
0 \leq \eta \leq \beta}} \sup _{z \in \mathbb{R}^{d}} \int_{\mathbb{R}^{d}}\left|\partial_{\xi}^{\eta}\left(\sigma^{\gamma}\left(z, T_{k} \xi\right)\right) \| \partial_{\xi}^{\beta-\eta}\left(\partial_{\xi}^{\alpha} \varphi_{k}\left(T_{k} \xi\right)\right)\right| d \xi\langle x\rangle_{B}^{-J} .
\end{aligned}
$$

Let us take a closer look at $\left|\partial_{\xi}^{\beta-\eta}\left(\partial_{\xi}^{\alpha} \varphi_{k}\left(T_{k} \xi\right)\right)\right|$. Put $\mu_{k}(\xi)=\varphi_{k}\left(T_{k} \xi\right)$. It is easy to verify that $\left|\partial_{\xi}^{\beta} \mu_{k}(\xi)\right| \leq C_{\beta} \chi_{Q}(\xi)$ where $Q \subset \mathbb{R}^{d}$ is a fixed compact set independent of $k$, see Equation (4.4). Notice that the chain rule yields

$$
\partial_{\xi}^{\alpha} \varphi_{k}\left(T_{k} \xi\right)=\sum_{|\beta|=|\alpha|} b_{\beta} \partial_{\xi}^{\beta} \mu_{k}(\xi)
$$

where $b_{\beta}$ are monomials of degree $|\beta|$ in the entries of $\delta_{h\left(\xi_{k}\right)^{-1}}$. Now, since $h(\xi) \geq \varepsilon_{0}>0$, each entry is uniformly bounded, and we obtain

$$
\left|\partial_{\xi}^{\beta-\eta}\left(\partial_{\xi}^{\alpha} \varphi_{k}\left(T_{k} \xi\right)\right)\right| \leq C \chi_{Q}(\xi) .
$$

Next, an estimate similar to the one in the proof of Proposition 7.6 (using (7.2) instead of (7.4)), gives that

$$
\left|\partial_{\xi}^{\eta}\left(\sigma^{\gamma}\left(z, T_{k} \xi\right)\right)\right| \leq C_{\eta}|\sigma|_{|\eta|, K}^{(0)} h\left(\xi_{k}\right)^{\alpha_{2}|\eta|} w\left(T_{k} \xi\right)^{-\rho|\eta|} .
$$

Since $h(\xi)^{\alpha_{2}} \leq w(\xi)^{\rho}$, and $w$ is a moderate function, we obtain

$$
\left|\partial_{\xi}^{\eta}\left(\sigma^{\gamma}\left(z, T_{k} \xi\right)\right)\right| \leq C_{\eta}|\sigma|_{|\eta|, K}^{(0)} \quad \text { for all } \xi \in Q .
$$

Finally, using the estimates (7.9) and (7.10) in (7.8) yields

$$
\begin{aligned}
& F\left(\delta_{h\left(\xi_{k}\right)}^{-\top} x\right) \\
& \quad \leq C^{\prime \prime}\left|\delta_{h\left(\xi_{k}\right)}^{\top}\right| \sum_{\substack{|\beta| \leq L \\
0 \leq \eta \leq \beta}}|\sigma|_{M, K}^{(0)} \int_{\mathbb{R}^{d}} \chi_{Q}(\xi) d \xi\langle x\rangle_{B}^{-J} \leq C^{\prime \prime \prime}\left|\delta_{h\left(\xi_{k}\right)}^{\top}\right| \cdot|\sigma|_{M, K}^{(0)}\langle x\rangle_{B}^{-J},
\end{aligned}
$$


which proves (a).

Let us turn to (b). Pick $J>m+\nu$ in (a). Then we have

$$
\begin{aligned}
I & =\int_{\mathbb{R}^{d}} F(x)\langle x\rangle_{B}^{m} d x \\
& \leq C^{\prime}|\sigma|_{M, K}^{(0)}\left|\delta_{h\left(\xi_{k}\right)}^{\top}\right| \int_{\mathbb{R}^{d}}\left\langle\delta_{h\left(\xi_{k}\right)}^{\top} x\right\rangle_{B}^{-J}\langle x\rangle_{B}^{m} d x \\
& =C^{\prime}|\sigma|_{M, K}^{(0)} \int_{\mathbb{R}^{d}}\langle x\rangle_{B}^{-J}\left\langle\delta_{h\left(\xi_{k}\right)}^{-\top} x\right\rangle_{B}^{m} d x
\end{aligned}
$$

However, $\left\langle\delta_{h\left(\xi_{k}\right)}^{-\top} x\right\rangle_{B} \leq C\langle x\rangle_{B}$ since $h\left(\xi_{k}\right) \geq \varepsilon_{0}>0$ so we obtain

$$
I \leq C^{\prime}|\sigma|_{M, K}^{(0)} \int_{\mathbb{R}^{d}}\langle x\rangle_{B}^{-J+m} d x \leq \tilde{C}|\sigma|_{M, K}^{(0)},
$$

since $J>\nu+m$. This concludes the proof.

We now turn to the proof of Theorem 7.4. For convenience, we use the notation

$$
\rho(z, D) f(x):=\frac{1}{(2 \pi)^{d / 2}} \int_{\mathbb{R}^{d}} \rho(z, \xi) \hat{f}(\xi) e^{i x \cdot \xi} d \xi, \quad x, z \in \mathbb{R}^{d},
$$

in the proof.

Proof of Theorem 7.4. Let $\left\{\varphi_{k}\right\}_{k \in \mathbb{N}}$ be the BAPU defined in (4.3) associated with the family $\left\{\delta_{h\left(\xi_{k}\right)} \cdot+\xi_{k}\right\}$ of affine transformations generated by $h$. From the facts that $\mathcal{J}^{-a} F_{p, q}^{s}(h, w)=F_{p, q}^{s+a}(h, w), \sigma(x, D) \mathcal{J}^{a} \in$ $\mathrm{Op} S_{w ; \rho}^{b+a}$ and $\mathcal{J}^{a} \sigma(x, D) \in \mathrm{Op} S_{w ; \rho}^{b+a}$ when $\sigma \in S_{w ; \rho}^{b}$, see Theorem 7.8, we deduce that it suffices to consider the case $b=0$ and $s>s_{0}$, where $s_{0}$ is chosen sufficiently large to ensure that $\left\{w\left(\xi_{k}\right)^{-\left(s_{0}-2 \rho \nu / \alpha_{2}\right)}\right\} \in \ell_{1}$ (notice that the lower bound in (7.3) guarantees the existence of $s_{0}$, see also Remark 5.8). It also suffices to prove that $\|\sigma(x, D) f\|_{F_{p, q}^{s}(h)} \leq C\|f\|_{F_{p, q}^{s}(h)}$ for $f \in \mathcal{S}\left(\mathbb{R}^{d}\right)$ since $\mathcal{S}\left(\mathbb{R}^{d}\right)$ is dense in $F_{p, q}^{s}(h, w)$.

Now suppose $\sigma \in S_{w ; \rho}^{0}$ and $s>s_{0}$. Notice that for $g \in \mathcal{S}\left(\mathbb{R}^{d}\right)$,

$$
\begin{aligned}
{\left[\varphi_{k}(D) g\right](x) } & =(2 \pi)^{-d / 2} \int_{\mathbb{R}^{d}} e^{i x \cdot \xi} \varphi_{k}(\xi) \hat{g}(\xi) d \xi \\
& =(2 \pi)^{-d / 2} \int_{\mathbb{R}^{d}} \hat{\varphi}_{k}(y) g(x+y) d y .
\end{aligned}
$$

Let $\sigma^{\gamma}(x, \xi):=\partial_{x}^{\gamma} \sigma(x, \xi)$. We obtain for any $K \in \mathbb{N}$

$$
\sigma(x+y, D) f(x+y)=(2 \pi)^{-d / 2} \int_{\mathbb{R}^{d}} e^{i(x+y) \cdot \xi} \sigma(x+y, \xi) \hat{f}(\xi) d \xi
$$




$$
\begin{aligned}
=(2 \pi)^{-d / 2} & \sum_{|\gamma| \leq K-1} \frac{y^{\gamma}}{\gamma !} \int_{\mathbb{R}^{d}} e^{i(x+y) \cdot \xi} \sigma^{\gamma}(x, \xi) \hat{f}(\xi) d \xi \\
& +(2 \pi)^{-d / 2} \sum_{|\gamma|=K} \frac{y^{\gamma}}{\gamma !} \int_{\mathbb{R}^{d}} e^{i(x+y) \cdot \xi} \int_{0}^{1}(1-\tau)^{K-1} \sigma^{\gamma}(x+\tau y, \xi) \hat{f}(\xi) d \tau d \xi
\end{aligned}
$$

$:=T(x, y)+R(x, y)$,

where we have expanded $\sigma(x+y, \xi)$ in a Taylor series around $x$. Using (7.12) in (7.11), we obtain

$$
\begin{aligned}
& \varphi_{k}(D) \sigma(x, D) f(x) \\
& \quad=(2 \pi)^{-d / 2} \int_{\mathbb{R}^{d}} \hat{\varphi}_{k}(y) T(x, y) d y+(2 \pi)^{-d / 2} \int_{\mathbb{R}^{d}} \hat{\varphi}_{k}(y) R(x, y) d y .
\end{aligned}
$$

We estimate each of the two terms separately. First we consider the term with $T(x, y)$. We have,

$$
\begin{aligned}
\int_{\mathbb{R}^{d}} \hat{\varphi}_{k}(y) T(x, y) d y= & (2 \pi)^{-d / 2} \int_{\mathbb{R}^{d}} \hat{\varphi}_{k}(y) \sum_{|\gamma| \leq K-1} \frac{y^{\gamma}}{\gamma !} \\
& \times \int_{\mathbb{R}^{d}} e^{i(x+y) \cdot \xi} \sigma^{\gamma}(x, \xi) \hat{f}(\xi) d \xi d y \\
= & (2 \pi)^{-d / 2} \sum_{|\gamma| \leq K-1} \frac{1}{\gamma !} \int_{\mathbb{R}^{d}} e^{i x \cdot \xi} \sigma^{\gamma}(x, \xi) \hat{f}(\xi) \\
& \times \int_{\mathbb{R}^{d}} e^{i y \cdot \xi} \hat{\varphi}_{k}(y) y^{\gamma} d y d \xi \\
= & \sum_{|\gamma| \leq K-1} \frac{C_{\gamma}}{\gamma !} \int_{\mathbb{R}^{d}} e^{i x \cdot \xi} \sigma^{\gamma}(x, \xi) \partial_{\xi}^{\gamma} \varphi_{k}(\xi) \hat{f}(\xi) d \xi .
\end{aligned}
$$

Using the fact that $\varphi_{k}^{*}(\xi)=1$ on $\operatorname{supp}\left(\varphi_{k}\right)$, and the relation $(\hat{f} \hat{g})^{\vee}=f * g$, we get

$$
\begin{aligned}
& \left|\int_{\mathbb{R}^{d}} e^{i x \cdot \xi} \sigma^{\gamma}(x, \xi) \partial_{\xi}^{\gamma} \varphi_{k}(\xi) \hat{f}(\xi) d \xi\right| \\
& \quad=\left|\int_{\mathbb{R}^{d}} e^{i x \cdot \xi}\left(\sigma^{\gamma}(x, \xi) \partial_{\xi}^{\gamma} \varphi_{k}(\xi)\right)\left(\varphi_{k}^{*}(\xi) \hat{f}(\xi)\right) d \xi\right| \\
& \quad \leq \int_{\mathbb{R}^{d}}\left|\left(\sigma^{\gamma}(x, \cdot) \partial_{\xi}^{\gamma} \varphi_{k}\right)^{\vee}(y)\right|\left|\varphi_{k}^{*}(D) f(x-y)\right| d y \\
& \quad \leq \int_{\mathbb{R}^{d}} \sup _{z \in \mathbb{R}^{d}}\left|\left(\sigma^{\gamma}(z, \cdot) \partial_{\xi}^{\gamma} \varphi_{k}\right)^{\vee}(y)\right|\left|\varphi_{k}^{*}(D) f(x-y)\right| d y .
\end{aligned}
$$


Using the estimates (a) and (b) from Lemma 7.9 we may apply Lemma 3.1 to conclude that

$$
\left|\int_{\mathbb{R}^{d}} e^{i x \cdot \xi} \sigma^{\gamma}(x, \xi) \partial_{\xi}^{\gamma} \varphi_{k}(\xi) \hat{f}(\xi) d \xi\right| \leq C|\sigma|_{L, K}^{(0)} M^{B}\left(\varphi_{k}^{*}(D) f\right)(x),
$$

with $C<\infty$ independent of $k$ and $f$, and where $L>(m+\nu)$. Finally, combining (7.14) and (7.15), the Fefferman-Stein inequality implies

$$
\left\|\left\{w\left(\xi_{k}\right)^{s} \int_{\mathbb{R}^{d}} \hat{\varphi}_{k}(y) T(\cdot, y) d y\right\}_{k}\right\|_{L_{p}\left(\ell_{q}\right)} \leq C|\sigma|_{L, K}^{(0)}\|f\|_{F_{p, q}^{s}(h)} .
$$

Now we turn to the second term in (7.13). We let $\Theta_{k}(\xi)=\varphi_{k}\left(T_{k} \xi\right)$, where $T_{k}=\delta_{h\left(\xi_{k}\right)}++\xi_{k}$. Put $t_{k}=h\left(\xi_{k}\right)$, and notice that $\hat{\Theta}_{k}(y)=$ $\left|\delta_{t_{k}}\right|^{-1} \hat{\varphi}_{k}\left(\delta_{t_{k}}^{-\top} y\right) e^{-i y \cdot \delta_{t_{k}}^{-1} \xi_{k}}$ from which we obtain

$$
\int_{\mathbb{R}^{d}} \hat{\varphi}_{k}(y) R(x, y) d y=\int_{\mathbb{R}^{d}} \hat{\Theta}_{k}(y) R\left(x, \delta_{t_{k}}^{-\top} y\right) e^{-i \xi_{k} \cdot y} d y .
$$

We have the estimate $\left|\left(\delta_{t_{k}}^{-\top} y\right)^{\gamma}\right| \leq c\left|\delta_{t_{k}}^{-\top} y\right|^{|\gamma|} \leq c^{\prime} t_{k}^{-|\gamma|}\langle y\rangle_{B}^{\alpha_{2}|\gamma|}, \forall y \in \mathbb{R}^{d}$ (since $\alpha_{1}=1$ ). Hence,

$$
\begin{aligned}
&\left|\int_{\mathbb{R}^{d}} \hat{\varphi}_{k}(y) R(x, y) d y\right| \\
& \leq \mid \mid \sum_{|\gamma|=K} \frac{1}{\gamma !} \int_{\mathbb{R}^{d}}\left(\delta_{t_{k}}^{-\top} y\right)^{\gamma} \hat{\Theta}_{k}(y) \int_{\mathbb{R}^{d}} e^{i\left(x+\delta_{t_{k}}^{-\top} y\right) \cdot \xi} \\
& \times \int_{0}^{1}(1-\tau)^{K-1} \sigma^{\gamma}\left(x+\tau \delta_{t_{k}}^{-\top} y, \xi\right) \hat{f}(\xi) d \tau d \xi e^{-i y \cdot \xi_{k}} d y \mid \\
& \leq C t_{k}^{-K} \sum_{|\gamma|=K} \int_{\mathbb{R}^{d}}\langle y\rangle_{B}^{K \alpha_{2}}\left|\hat{\Theta}_{k}(y)\right| \mid \int_{0}^{1}(1-\tau)^{K-1} \\
& \times \int_{\mathbb{R}^{d}} e^{i\left(x+\delta_{t_{k}}^{-\top} y\right) \cdot \xi} \sigma^{\gamma}\left(x+\tau \delta_{t_{k}}^{-\top} y, \xi\right) \hat{f}(\xi) d \xi d \tau \mid d y .
\end{aligned}
$$

Fix $\theta \in(1,2)$. We use the estimate $\left|\hat{\Theta}_{k}(y)\right| \leq C\langle y\rangle_{B}^{-K \alpha_{2}-\nu-1-\theta \nu}$ given by (4.5) to obtain

$$
\begin{aligned}
& C^{\prime} t_{k}^{-K} \sum_{|\gamma|=K} \int_{\mathbb{R}^{d}} \frac{\langle y\rangle_{B}^{-\nu-1}}{\langle y\rangle_{B}^{\theta \nu}} \sup _{z \in \mathbb{R}^{d}}\left|\left[\sigma^{\gamma}(z, D) f\right]\left(x+\delta_{t_{k}}^{-\top} y\right)\right| d y \\
& \quad=C^{\prime} t_{k}^{-K} \sum_{|\gamma|=K} \int_{\mathbb{R}^{d}}\langle y\rangle_{B}^{-\nu-1} \sup _{z \in \mathbb{R}^{d}} \frac{\left|\left[\sigma^{\gamma}(z, D) f\right]\left(x+\delta_{t_{k}}^{-\top} y\right)\right|}{\langle y\rangle_{B}^{\theta \nu}} d y
\end{aligned}
$$




$$
\begin{aligned}
& \leq C^{\prime} t_{k}^{-K} \sum_{|\gamma|=K} \int_{\mathbb{R}^{d}}\langle y\rangle_{B}^{-\nu-1} \sup _{z, v \in \mathbb{R}^{d}} \frac{\left|\left[\sigma^{\gamma}(z, D) f\right](x+v)\right|}{\left\langle\delta_{t_{k}}^{\top} v\right\rangle_{B}^{\theta \nu}} d y \\
& \leq C^{\prime \prime} t_{k}^{-K} \sum_{|\gamma|=K} \sup _{z, v \in \mathbb{R}^{d}} \frac{\left|\left[\sigma^{\gamma}(z, D) f\right](x+v)\right|}{\langle v\rangle_{B}^{\theta \nu}}
\end{aligned}
$$

since $\left\langle\delta_{t_{k}}^{\top} v\right\rangle_{B} \geq c^{\prime}\langle v\rangle_{B}$ due to the assumption that $t_{k} \geq \varepsilon_{0}>0$. We have,

$$
\begin{aligned}
S(x) & :=\left(\sum_{k}\left(w\left(\xi_{k}\right)^{s} \int_{\mathbb{R}^{d}} \hat{\varphi}_{k}(y) R(x, y) d y\right)^{q}\right)^{1 / q} \\
& \leq C\left(\sum_{k}\left(w\left(\xi_{k}\right)^{s} t_{k}^{-K} \sum_{|\gamma|=K^{\prime}} \sup _{z, v \in \mathbb{R}^{d}} \frac{\left|\left[\sigma^{\gamma}(z, D) f\right](x+v)\right|}{\langle v\rangle_{B}^{\theta \nu}}\right)^{q}\right)^{1 / q} \\
& \leq C \sum_{|\gamma|=K} \sup _{z, v \in \mathbb{R}^{d}} \frac{\left|\left[\sigma^{\gamma}(z, D) f\right](x+v)\right|}{\langle v\rangle_{B}^{\theta \nu}}\left(\sum_{k}\left(t_{k}^{s \alpha_{2} / \kappa} t_{k}^{-K}\right)^{q}\right)^{1 / q} \\
& \leq C^{\prime \prime} \sum_{|\gamma|=K} \sum_{m} \sup _{z, v \in \mathbb{R}^{d}} \frac{\left|\left[\sigma^{\gamma}(z, D) \varphi_{m}(D) f\right](x+v)\right|}{\langle v\rangle_{B}^{\theta \nu}}
\end{aligned}
$$

where we used the fact that $w(\xi) \leq\langle\xi\rangle \leq\langle\xi\rangle_{A}^{\alpha_{2}} \leq h(\xi)^{\alpha_{2} / \kappa}$, and that $\sum_{k} t_{k}^{\left(s \alpha_{2} / \kappa-K\right) q}<\infty$ provided $K$ is sufficiently large. We estimate the term $A_{m}(z, x):=\left|\left[\sigma^{\gamma}(z, D) \varphi_{m}(D) f\right](x)\right|$. Let $f_{m}(x):=\left[\varphi_{m}^{*}(D) f\right](x)$. We have

$$
\begin{aligned}
A_{m}(z, x+v) & =\left|\int_{\mathbb{R}^{d}}\left(\sigma^{\gamma}(z, \cdot) \varphi_{m}(\cdot)\right)^{\vee}(x+v-y) f_{m}(y) d y\right| \\
& \leq \int_{\mathbb{R}^{d}}\left|\left(\sigma^{\gamma}(z, \cdot) \varphi_{m}(\cdot)\right)^{\vee}(x+v-y)\right|\left|f_{m}(y)\right| d y \\
& \leq \sup _{u \in \mathbb{R}^{d}} \frac{\left|f_{m}(u)\right|}{\langle x-u\rangle_{B}^{\theta \nu}} \int_{\mathbb{R}^{d}}\left|\left(\sigma^{\gamma}(z, \cdot) \varphi_{m}(\cdot)\right)^{\vee}(x+v-y)\right|\langle x-y\rangle_{B}^{\theta \nu} d y .
\end{aligned}
$$

Now, by Lemma 2.3, $\langle x-y\rangle_{B}^{\theta \nu} \leq\left(c^{\prime}\right)^{\theta \nu}\langle x-y+v\rangle_{B}^{\theta \nu}\langle v\rangle_{B}^{\theta \nu}$, so

$$
\begin{aligned}
\sup _{z, v \in \mathbb{R}^{d}} \frac{A_{m}(z, x+v)}{\langle v\rangle_{B}^{\theta \nu}} & \leq C \sup _{v \in \mathbb{R}^{d}} \frac{\left|f_{m}(x-v)\right|}{\langle v\rangle_{B}^{\theta \nu}} \sup _{z \in \mathbb{R}^{d}} \int_{\mathbb{R}^{d}}\left|\left(\sigma^{\gamma}(z, \cdot) \varphi_{m}(\cdot)\right)^{\vee}(u)\right|\langle u\rangle_{B}^{\theta \nu} d u \\
& \leq C^{\prime} \sup _{v \in \mathbb{R}^{d}} \frac{\left|f_{m}(x-v)\right|}{\langle v\rangle_{B}^{\theta \nu}}|\sigma|_{L, K}^{(0)},
\end{aligned}
$$


where we used Lemma 7.9 in the last step. Using the estimate $\left\langle\delta_{t_{m}}^{\top} v\right\rangle_{B} \leq$ $C t_{m}\langle v\rangle_{B}$ we finally obtain

$S(x) \leq C|\sigma|_{L, K}^{(0)} \sum_{m} \frac{t_{m}^{\theta \nu}}{t_{m}^{\theta \nu}} \sup _{v \in \mathbb{R}^{d}} \frac{\left|f_{m}(x-v)\right|}{\langle v\rangle_{B}^{\theta \nu}} \leq C|\sigma|_{L, K}^{(0)} \sum_{m} t_{m}^{\theta \nu} \sup _{v \in \mathbb{R}^{d}} \frac{\left|f_{m}(x-v)\right|}{\left\langle\delta_{t_{m}}^{\top} v\right\rangle_{B}^{\theta \nu}}$

and by Proposition 3.4,

$$
\|S(x)\|_{L_{p}} \leq C|\sigma|_{L, K}^{(0)}\left\|\left\{t_{m}^{\theta \nu} f_{m}\right\}_{m}\right\|_{L_{p}\left(\ell_{1}\right)}=C|\sigma|_{L, K}^{(0)}\|f\|_{F_{p, 1}^{\tilde{s}(h, w)}},
$$

where $\tilde{s}=\frac{\rho \theta \nu}{\alpha_{2}}$. Since $\left\{w\left(\xi_{k}\right)^{-(s-\tilde{s})}\right\} \in \ell_{1}$ we deduce from Proposition 5.7 that

$$
\|f\|_{F_{p, 1}^{\tilde{s}}(h, w)} \leq C\|f\|_{F_{p, q}^{s}(h, w)}
$$

which concludes the proof.

7.1. Some remarks. The proof of Theorem 7.4 can be modified (and simplified) in a straightforward way to prove the corresponding result for modulation spaces. We leave the details for the reader.

Theorem 7.10. Given $\sigma \in S_{w ; \rho}^{b}\left(\mathbb{R}^{d} \times \mathbb{R}^{d}\right), b \in \mathbb{R}, \rho \in(0,1]$. Suppose the moderate weight $h$ satisfies

$$
C_{1}\langle\xi\rangle_{A}^{\kappa} \leq h(\xi) \leq C_{2} w(\xi)^{\rho / \alpha_{2}} \quad \forall \xi \in \mathbb{R}^{d},
$$

for some $\kappa>0$. Then $\sigma(x, D)$ extends to a bounded operator

$$
\sigma(x, D): M_{p, q}^{s+b}(h, w) \rightarrow M_{p, q}^{s}(h, w) .
$$

for any $s \in \mathbb{R}$, and $p, q \in[1, \infty)$.

For homogeneous symbols $\sigma \in S_{w ; \rho}^{b}$ one can improve Theorems 7.4 and 7.10. Suppose $\sigma \in C^{\infty}\left(\mathbb{R}^{d} \times \mathbb{R}^{d}\right)$ and there exists a constant $b \in \mathbb{R}$ such that $\sigma\left(x, \delta_{t} \xi\right)=t^{b} \sigma(x, \xi)$ for all $t \geq 1$ and $\xi \in \mathbb{R}^{d}$. One can verify that $\sigma \in S_{w ; \rho}^{b}$ using the homogeneity. Similar arguments as in the proof of Theorem 7.4, and a modification of Lemma 7.9, show that $\sigma(x, D)$ extends to a bounded operator

$$
\sigma(x, D): F_{p, q}^{s}(h, w) \rightarrow F_{p, q}^{s-b}(h, w),
$$

provided $C_{1}\langle\xi\rangle_{A}^{\kappa} \leq h(\xi) \leq C_{2} w(\xi)^{\rho}$ for some $\kappa>0$. An analogous result holds in the modulation case. Hence, we obtain a boundedness result for a much larger class of spaces $F_{p, q}^{s}(h, w)\left[M_{p, q}^{s}(h, w)\right]$. We leave the details for the reader.

Another way to strengthen Theorem 7.4 is to restrict the analysis to diagonal matrices $A$. Suppose $A=\operatorname{diag}\left(a_{1}, \ldots, a_{d}\right)$, and put $\mathbf{a}=$ 
$\left(a_{1}, \ldots, a_{d}\right)$. For $b \in \mathbb{R}$ and $\rho>0$ define the Hörmander class $S_{w ; \rho}^{\mathbf{a}, b}\left(\mathbb{R}^{d} \times \mathbb{R}^{d}\right)$ as the family of functions $\sigma \in C^{\infty}\left(\mathbb{R}^{d} \times \mathbb{R}^{d}\right)$ satisfying

$$
|\sigma|_{N, M}^{(b)}:=\max _{|\alpha| \leq N,|\beta| \leq M} \sup _{x, \xi \in \mathbb{R}^{d}} w(\xi)^{\rho \mathbf{a} \cdot \alpha-b}\left|\partial_{\xi}^{\alpha} \partial_{x}^{\beta} \sigma(x, \xi)\right|<\infty,
$$

for $M, N \in \mathbb{N}$. Then, using similar arguments as in the proof of Theorem 7.4, it can be verified that any $\sigma \in S_{w ; \rho}^{\mathbf{a}, b}$ extends to a bounded operator $\sigma(x, D): F_{p, q}^{s}(h, w) \rightarrow F_{p, q}^{s-b}(h, w)$ provided $C_{1}\langle\xi\rangle_{A}^{\kappa} \leq h(\xi) \leq C_{2} w(\xi)^{\rho}$ for some $\kappa>0$.

7.2. Elliptic operators. We conclude this section with a brief discussion of elliptic symbols. We have the following definition.

Definition 7.11. A symbol $\sigma \in S_{w ; \rho}^{b}$ is called $w$-elliptic if there exist $C, M>0$ such that $|\sigma(x, \xi)| \geq C w(\xi)^{b}$ for $\langle\xi\rangle \geq M$.

We let $S_{w ; \rho}^{-\infty}:=\cap_{m \in \mathbb{R}} S_{w ; \rho}^{m}$. The following result on elliptic symbols is well-known and the proof can be found in, e.g., [27].

Theorem 7.12. Suppose $\sigma \in S_{w ; \rho}^{b}$ is $w$-elliptic and there exist $C, \kappa>0$ such that $w(\xi) \geq C\langle\xi\rangle^{\kappa}$ for $\xi \in \mathbb{R}^{d}$. Then there exists $\tau \in S_{w ; \rho}^{-b}$ such that $I-\sigma(x, D) \tau(x, D)$ and $I-\tau(x, D) \sigma(x, D)$ are both in $O p\left(S_{w ; \rho}^{-\infty}\right)$.

Now we put $F_{p, q}^{-\infty}(h, w)=\cup_{s \in \mathbb{R}} F_{p, q}^{s}(h, w)$. Using Theorem 7.12 and the previous results we have

Proposition 7.13. Suppose $\sigma \in S_{w ; \rho}^{b}$ is $w$-elliptic and assume that the moderate weight $h$ satisfies (7.3). If $f \in F_{p, q}^{-\infty}(h, w)$ and $\sigma(\cdot, D) f \in$ $F_{p, q}^{s}(h, w)$ for some $s \in \mathbb{R}$, then $f \in F_{p, q}^{s+b_{0}}(h, w)$.

Proof. Let $S=\sigma(\cdot, D)$, and let $T=\tau(\cdot, D)$ be as in Theorem 7.12. Notice that $f=T(S f)+(I-T S) f$. By Theorem 7.4, T maps $F_{p, q}^{s}(h, w)$ to $F_{p, q}^{s+b}(h, w)$ and $(I-T S)$ maps $F_{p, q}^{-\infty}(h, w)$ to $F_{p, q}^{s+b}(h, w)$.

\section{Examples}

This final section is devoted to a number of examples of T-L type spaces. For simplicity we will consider the moderate weight function defined in Example 4.6.

8.1. Isotropic spaces. Let us consider the isotropic case. We put $A=$ $\operatorname{diag}(1,1, \ldots, 1)$, and $|\cdot|_{A}$ is the Euclidean norm with $\langle\cdot\rangle$ the standard bracket on $\mathbb{R}^{d}$. We define the moderate function $h_{\alpha}(\xi)=\langle\xi\rangle^{\alpha}$, where 
$\alpha \in[0,1]$ is fixed. Observe that any covering ball $B$ in $\mathbb{R}^{d}$ associated with $h_{\alpha}$ satisfies the following simple geometric rule:

$$
\xi \in B \Rightarrow\langle\xi\rangle^{\alpha d} \asymp|B| .
$$

For the weight $h_{\alpha}, F_{p, q}^{\beta}\left(h_{\alpha},\langle\cdot\rangle\right)$ and $M_{p, q}^{\beta}\left(h_{\alpha},\langle\cdot\rangle\right)$ are well-defined spaces. It turns out that the spaces $M_{p, q}^{\beta}\left(h_{\alpha},\langle\cdot\rangle\right)$ are the so-called $\alpha$-modulation spaces $M_{p, q}^{\beta, \alpha}\left(\mathbb{R}^{d}\right)$ introduced by Gröbner [24], while $F_{p, q}^{\beta}\left(h_{\alpha},\langle\cdot\rangle\right)$ is a new family of spaces (for $\alpha<1$ ). Let us consider the spaces in more detail.

8.1.1. The case $\alpha=1$. First we consider the case $\alpha=1$, where (8.1) corresponds to a "dyadic" cover which gives us a classical T-L space. Let $E_{2}=\{ \pm 1, \pm 2\}, E_{1}:=\{ \pm 1\}$, and $E:=E_{2}^{d} \backslash E_{1}^{d}$. For each $k \in E$, and $j \in \mathbb{N}$ define $b_{j, k}:=2^{j}\left(v\left(k_{1}\right), \ldots, v\left(k_{d}\right)\right)$, where

$$
v(k)=\operatorname{sgn}(k) \cdot \begin{cases}1 / 2 & \text { for } k=1 \\ 3 / 2 & \text { for } k=2\end{cases}
$$

Suppose $\mathcal{T}=\left\{4 I, T_{j, k}\right\}_{j \in \mathbb{N}, k \in E}$ is given by $T_{j, k} \xi=2^{j} \xi+b_{j, k}$ and let $Q \subset \mathbb{R}^{d}$ be an open cube with center 0 and side length $r>1 / 2$. Then $\left\{T_{j, k} Q\right\}_{j \in \mathbb{N}, k \in E}$ is a structured admissible covering of $\mathbb{R}^{d}$ of the type used in Section 5. Figure 1 illustrate this covering for $d=2$.

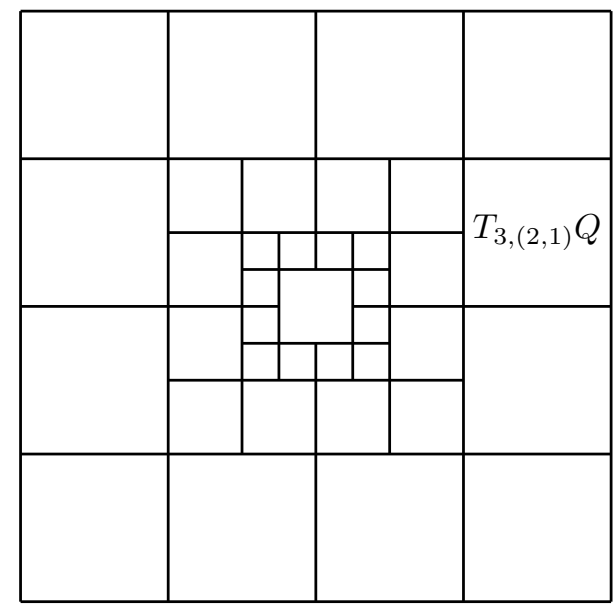

Figure 1. A decomposition of the frequency plane using the structured family $\mathcal{T}$ from Section 8.1.1 on a fixed cube $Q$ 
Using $\mathcal{T}$ it can be verified that $F_{p, q}^{\beta}\left(h_{1},\langle\cdot\rangle\right)$ is a T-L space. In fact $F_{p, q}^{\beta}\left(h_{1},\langle\cdot\rangle\right)=F_{p, q}^{\beta}\left(\mathbb{R}^{d}\right)$, see e.g. [36] for details. In this case, one can also verify that $M_{p, q}^{\beta}\left(h_{1},\langle\cdot\rangle\right)=B_{p, q}^{\beta}\left(\mathbb{R}^{d}\right)$ is a classical Besov space.

8.1.2. The case $0 \leq \boldsymbol{\alpha}<\mathbf{1}$. This case is more interesting as we obtain new families of spaces. Define $b_{k}=k|k|^{\alpha /(1-\alpha)}, k \in \mathbb{Z}^{d} \backslash\{0\}$, and let $\mathcal{T}=\left\{T_{k}\right\}_{k \in \mathbb{Z}^{d} \backslash\{0\}}$ be given by $T_{k} \xi=|k|^{\alpha /(1-\alpha)} \xi+b_{k}$. This type of "polynomial" covering was first considered by Päivärinta and Somersalo in [30] to study pseudodifferential operators, and Gröbner [24] used such coverings to define $\alpha$-modulation spaces. It is not difficult to verify that $M_{p, q}^{\beta}\left(h_{\alpha},\langle\cdot\rangle\right)=M_{p, q}^{\beta, \alpha}\left(\mathbb{R}^{d}\right)$, and consequently $F_{p, q}^{\beta}\left(h_{\alpha},\langle\cdot\rangle\right)$ can be considered the T-L equivalent of the $\alpha$-modulation spaces. In [5], the authors introduced so-called $\alpha$-Triebel-Lizorkin space in the one dimensional case. One can verify that the $\alpha$-Triebel-Lizorkin scale equals the T-L type space $F_{p, q}^{\beta}\left(h_{\alpha},\langle\cdot\rangle\right)$. However, the authors were not able to prove in [5] that these spaces are independent of the particular BAPU. This important property now follows directly from Proposition 5.3.

In this case we have an explicitly given covering, so we can also write out the norm equivalence given by (6.6). Let $\left\{\eta_{k, n}\right\}$ be the tight frame for $F_{p, q}^{\beta}\left(h_{\alpha},\langle\cdot\rangle\right)$ given in Section 6 . We have

$$
\|f\|_{F_{p}^{\beta}, q\left(h_{\alpha},\langle\cdot\rangle\right)} \asymp\left\|\left\langle\langle k\rangle^{\frac{s}{1-\alpha}+\frac{1}{2} \frac{\alpha d}{1-\alpha}}\left(\sum_{n \in \mathbb{Z}^{d}}\left|\left\langle f, \eta_{k, n}\right\rangle\right|^{q} \chi_{Q(k, n)}\right)^{1 / q}\right\}_{k}\right\|_{L_{p}\left(\ell_{q}\right)},
$$

where $Q(k, n)$ is given by (6.3) with $T_{k}=|k|^{\alpha /(1-\alpha)} \cdot+b_{k}$.

8.1.3. Pseudo-differential operators on isotropic spaces. For $A=$ $\operatorname{diag}(1,1, \ldots, 1)$ we may take $\alpha_{2}=\alpha_{1}=1$. Then Theorem 7.4 says that any pseudo-differential operator $\sigma \in S_{\langle\cdot\rangle ; \rho}^{b}$ extends to a bounded operator

$$
\sigma(x, D): F_{p, q}^{\beta}\left(h_{\alpha},\langle\cdot\rangle\right) \rightarrow F_{p, q}^{\beta-b}\left(h_{\alpha},\langle\cdot\rangle\right)
$$

provided $0<\alpha \leq \rho$. For $\alpha=1$ this simplifies to a well-known boundedness result for T-L spaces [29, 10], and for $0 \leq \alpha<1$, it extends the authors result [4] for $\alpha$-modulation spaces to T-L type spaces. We also notice that with this particular choice of dilation and weights, Theorem 7.10 reproduces the main result in [4],

$$
\sigma(x, D): M_{p, q}^{\beta}\left(h_{\alpha},\langle\cdot\rangle\right) \rightarrow M_{p, q}^{\beta-b}\left(h_{\alpha},\langle\cdot\rangle\right),
$$

where $\sigma \in S_{\langle\cdot\rangle ; \rho}^{b}$ and $0<\alpha \leq \rho$. 
8.2. Anisotropic spaces. Now we consider the same construction as above but adapted to the anisotropic setting. For a real matrix $A$ with eigenvalues with positive real parts, we consider the anisotropic distance $|\cdot|_{A}$. Define $h_{\alpha}(\xi)=\langle\xi\rangle_{A}^{\alpha}$, which is moderate according to Lemma 4.5. We can use Proposition 5.3 to conclude that $h_{\alpha}$ determines a decomposition space (up to equivalent norms) for each choice of moderate weight $w$. In particular, the space $F_{p, q}^{\beta}\left(h_{\alpha},\langle\cdot\rangle_{A}\right)$ is well-defined. Also notice that for $A=\operatorname{diag}(1,1, \ldots, 1), h_{\alpha}$ is the regulation function considered in the isotropic case. In this particular case, we obtain the same isotropic spaces as in Section 8.1. In general, we obtain anisotropic versions of the spaces considered in Section 8.1.

8.2.1. The case $\alpha=1$. This case corresponds to anisotropic T-L spaces. In the restricted case where $A$ is a diagonal matrix $A=\operatorname{diag}\left(a_{1}, \ldots, a_{d}\right)$ it can can be verified that $F_{p, q}^{\beta}\left(h_{1},\langle\cdot\rangle_{A}\right)$ is an anisotropic T-L space of the type considered in e.g. $[39,14]$. In fact $F_{p, q}^{\beta}\left(h_{1},\langle\cdot\rangle_{A}\right)=F_{p, q}^{\mathbf{a}, \beta}\left(\mathbb{R}^{d}\right)$, where $\mathbf{a}=\left(a_{1}, \ldots, a_{d}\right)$.

Suppose $\mathcal{T}=\left\{\delta_{\left\langle\xi_{k}\right\rangle_{A}} \cdot+\xi_{k}\right\}_{k \in \mathbb{N}}$ is one of the equivalent structured covering given by Lemma 4.7 . The tight frame $\left\{\eta_{k, n}\right\}$ of Section 6 yields an atomic decomposition of $F_{p, q}^{\beta}\left(h_{1},\langle\cdot\rangle_{A}\right)$, and (6.6) gives a characterization of the (quasi-)norm on $F_{p, q}^{\beta}\left(h_{1},\langle\cdot\rangle_{A}\right)$.

8.2.2. The case $0 \leq \boldsymbol{\alpha}<\mathbf{1}$. Next we consider the case $0 \leq \alpha<1$, which corresponds to a family of spaces which we will call anisotropic $\alpha-\mathrm{T}-\mathrm{L}$ spaces. According to Proposition 5.3, $F_{p, q}^{\beta}\left(h_{\alpha},\langle\cdot\rangle_{A}\right)$ is well-defined. Lemma 4.7 tells us that there exists a structured covering associated with $h_{\alpha}$, but unlike the isotropic case, we do not (in general) know of any explicitly given structured covering. However, if $\mathcal{T}=\left\{\delta_{h_{\alpha}}\left(\xi_{k}\right) \cdot+\xi_{k}\right\}_{k \in \mathbb{N}}$ is one of the equivalent structured covering given by Lemma 4.7 , then the tight frame $\left\{\eta_{k, n}\right\}$ of Section 6 yields an atomic decomposition of $F_{p, q}^{\beta}\left(h_{\alpha},\langle\cdot\rangle_{A}\right)$, and (6.6) gives a discrete characterization of the (quasi-)norm on $F_{p, q}^{\beta}\left(h_{\alpha},\langle\cdot\rangle_{A}\right)$.

8.2.3. Pseudo-differential operators on anisotropic spaces. For a real $d \times d$ matrix $A$ with eigenvalues with real parts in the interval $\left(1, \alpha_{2}\right)$ and $\alpha \in[0,1]$, we consider the anisotropic space $F_{p, q}^{\beta}\left(h_{\alpha},\langle\cdot\rangle_{A}\right)$ from Section 8.2.2. In this case, Theorem 7.4 states that a pseudo-differential operator $\sigma \in S_{\langle\cdot\rangle_{A} ; \rho}^{b}$ extends to a bounded operator

$$
\sigma(x, D): F_{p, q}^{\beta}\left(h_{\alpha},\langle\cdot\rangle_{A}\right) \rightarrow F_{p, q}^{\beta-b}\left(h_{\alpha},\langle\cdot\rangle_{A}\right)
$$

provided $0<\alpha \leq \rho / \alpha_{2}$. We also mention that Theorem 7.10 gives

$$
\sigma(x, D): M_{p, q}^{\beta}\left(h_{\alpha},\langle\cdot\rangle_{A}\right) \rightarrow M_{p, q}^{\beta-b}\left(h_{\alpha},\langle\cdot\rangle_{A}\right)
$$


for $0<\alpha \leq \rho / \alpha_{2}$. As noticed in Section 7.1 there is a stronger result when $A$ is diagonal. More precisely, if $\sigma \in S_{\langle\cdot\rangle_{A} ; \rho}^{\mathbf{a}, b}, A=\operatorname{diag}\left(a_{1}, \ldots, a_{d}\right)$, then (8.2) holds true provided $0<\alpha \leq \rho$.

8.2.4. The heat operator. We conclude by considering the heat operator $L$ on $\mathbb{R}^{2}$ with symbol $\sigma(t, x)=i t+x^{2}$. Put $A=\operatorname{diag}(2,1)$, and let $w(t, x):=\langle(t, x)\rangle_{A}$. One verifies that $w(t, x) \asymp 1+|t|^{1 / 2}+|x|$ and $\sigma \in S_{\langle\cdot\rangle_{A}, 1}^{2}\left(\mathbb{R}^{2} \times \mathbb{R}^{2}\right)$. We obtain

$$
L: F_{p, q}^{\beta}\left(h_{\alpha},\langle\cdot\rangle_{A}\right) \rightarrow F_{p, q}^{\beta-2}\left(h_{\alpha},\langle\cdot\rangle_{A}\right)
$$

provided $0<\alpha \leq 1 / 2$. Notice that $L$ is $\langle\cdot\rangle_{A}$-elliptic and we can construct an approximate inverse for $L$ by letting $a(u, t, x)=\eta(t, x)\left(i t+x^{2}\right)^{-1}$, $u \in \mathbb{R}^{2}, t, x \in \mathbb{R}$, where $\eta$ is a smooth cut-off function that vanishes at the origin and equals 1 away from the origin. One can check directly that $a \in S_{\langle\cdot\rangle_{A}, 1}^{-2}\left(\mathbb{R}^{2} \times \mathbb{R}^{2}\right)$. Here we notice that $a$ is only contained in $S_{\langle\cdot\rangle, 1 / 2}^{-1}\left(\mathbb{R}^{2} \times \mathbb{R}^{2}\right)$ due to the slow decay of $a$ as $t \rightarrow \infty$. Of course, $a$ is not $\langle\cdot\rangle$-elliptic, only hypoelliptic.

\section{Appendix A. Some technical proofs}

Here we give some of the more technical proofs. In particular, we prove Proposition 3.3 and Proposition 5.2. We begin by proving Proposition 3.3.

Proof of Proposition 3.3. Put $v(x)=\left|\delta_{R}^{-1}\right| u\left(\delta_{R}^{-\top} x\right)$ and let $a=$ $\nu / r$. Then $\hat{v}(\xi)=\hat{u}\left(\delta_{R} \xi\right)$ so $\operatorname{supp}(\hat{v}) \subset \mathcal{B}_{A}(0,1)$. Moreover, we have $v^{*}(a, 1 ; x)=\left|\delta_{R}^{-1}\right| u^{*}\left(a, R ; \delta_{R}^{-\top} x\right)$ and $M_{r}^{B} v(x)=\left|\delta_{R}^{-1}\right| M_{r}^{B} u\left(\delta_{R}^{-\top} x\right)$ so we may assume that $R=1$.

We take $\varphi \in \mathcal{S}\left(\mathbb{R}^{d}\right)$ with $\hat{\varphi}=1$ on $\mathcal{B}_{A}(0,1)$, and write $u(x-z)=$ $C \int_{\mathbb{R}^{d}} u(y) \varphi(x-z-y) d y$. Hence

(A.1)

$\left|\frac{\partial u}{\partial x_{j}}(x-z)\right|=C\left|\int_{\mathbb{R}^{d}} u(y) \frac{\partial \varphi}{\partial x_{j}}(x-z-y) d y\right| \leq C \int_{\mathbb{R}^{d}}|u(y)|\langle x-y-z\rangle_{B}^{-\lambda} d y$,

for any $\gamma>0$. We divide (A.1) by $\langle z\rangle_{B}^{a}$ and use the estimate $\langle x-$ $y\rangle_{B}^{a} /\langle z\rangle_{B}^{a} \leq c^{a}\langle x-y-z\rangle_{B}^{a}$ from Lemma 2.3 to deduce that

$$
\sup _{z \in \mathbb{R}^{d}}\langle z\rangle_{B}^{-a}|\nabla u(x-z)| \leq C^{\prime} \sup _{z \in \mathbb{R}^{d}}\langle z\rangle_{B}^{-a}|u(x-z)| .
$$

Fix $0<\eta \leq 1$. Let $x, z \in \mathbb{R}^{d}$ and suppose $y \in \mathcal{B}_{B}(x-z, \eta)$. We have

$$
|u(x-z)|^{r} \leq C_{r}\left(|u(x-z)-u(y)|^{r}+|u(y)|^{r}\right) .
$$


By the mean value theorem, with $\alpha_{2}$ and $c_{2}$ given by (2.3),

$$
|u(x-z)-u(y)|^{r} \leq\left|\nabla u\left(p_{0}\right)\right|^{r}|x-z-y|^{r} \leq c_{2}^{-r / \alpha_{2}} C_{r}\left|\nabla u\left(p_{0}\right)\right|^{r} \eta^{r / \alpha_{2}},
$$

with $p_{0} \in L(x-z, y)$. We have $\left|x-z-p_{0}\right|_{B} \leq \eta \leq 1$ since $\mathcal{B}_{B}(x-z, \eta)$ is convex, and consequently,

$$
\begin{aligned}
& \left(\omega_{d}^{B}\right)^{-1} \eta^{-\nu} \int_{\mathcal{B}_{B}(x-z, \eta)}|u(x-z)|^{r} d y \\
& \leq C_{r} \eta^{r / \alpha_{2}} \sup _{w \in \mathcal{B}_{B}(x-z, 1)}|\nabla u(w)|^{r}+C_{r}\left(\omega_{d}^{B}\right)^{-1} \eta^{-\nu} \int_{\mathcal{B}_{B}(x-z, \eta)}|u(y)|^{r} d y .
\end{aligned}
$$

Thus,

$$
|u(x-z)| \leq \tilde{C} \eta^{1 / \alpha_{2}} \sup _{w \in \mathcal{B}_{B}(x-z, 1)}|\nabla u(w)|+\tilde{C} \eta^{-a}\left(\int_{\mathcal{B}_{B}(x-z, \eta)}|u(y)|^{r} d y\right)^{1 / r} .
$$

By the quasi-triangle inequality, we obtain

$$
\begin{aligned}
\int_{\mathcal{B}_{B}(x-z, \eta)}|u(y)|^{r} d y & \leq \int_{\mathcal{B}_{B}\left(x, c\left(1+|z|_{B}\right)\right)}|u(y)|^{r} d y \\
& \leq c^{\prime} \omega_{d}^{B}\left(1+|z|_{B}\right)^{\nu}\left(M_{r}^{B} u(x)\right)^{r} \\
& \leq c^{\prime \prime} \omega_{d}^{B}\langle z\rangle_{B}^{\nu}\left(M_{r}^{B} u(x)\right)^{r} .
\end{aligned}
$$

Also, if $w \in \mathcal{B}_{B}(x-z, 1)$,

$c\langle z\rangle_{B} \geq\langle x-w\rangle_{B}-c\langle x-w-z\rangle_{B} \geq\langle x-w\rangle_{B}-c^{2}\left(1+|x-w-z|_{B}\right) \geq\langle x-w\rangle_{B}-2 c^{2}$, and since $\langle z\rangle_{B} \geq 1$, we obtain $\langle z\rangle_{B} \geq \frac{1}{c+2 c^{2}}\langle x-w\rangle_{B}$. Finally,

$$
\begin{aligned}
\langle z\rangle_{B}^{-a}|u(x-z)| & \leq C \eta^{1 / \alpha_{2}} \sup _{w \in \mathcal{B}_{B}(x-z, 1)}\langle x-w\rangle_{B}^{-a}|\nabla u(w)|+C \eta^{-a} M_{r}^{B} u(x) \\
& \leq C \eta^{1 / \alpha_{2}} \sup _{w \in \mathbb{R}^{d}}\langle w\rangle_{B}^{-a}|\nabla u(x-w)|+C \eta^{-a} M_{r}^{B} u(x) \\
& \leq C^{\prime} C \eta^{1 / \alpha_{2}} u^{*}(a, 1 ; x)+C \eta^{-a} M_{r}^{B} u(x) .
\end{aligned}
$$

The result now follows by choosing $\eta<\min \left\{1,\left(2 C C^{\prime}\right)^{-\alpha_{2}}\right\}$.

Next we turn to a proof of Proposition 5.2 demonstrating that the spaces introduced in Section 5 are indeed quasi-Banach spaces.

Proof of Proposition 5.2. It is easy to see that the functions $\|\cdot\|_{F_{p, q}^{s}(h, w)}$ and $\|\cdot\|_{M_{p, q}^{s}(h, w)}$ given in Definition 5.1 are quasi-norms (norms if $p, q \geq 1$ ). Let us first prove that $\mathcal{S}\left(\mathbb{R}^{d}\right) \hookrightarrow F_{p, q}^{s}(h, w)\left[\right.$ resp. $\left.M_{p, q}^{s}(h, w)\right] \hookrightarrow \mathcal{S}^{\prime}\left(\mathbb{R}^{d}\right)$. 
Let $s_{0}$ be such that $w\left(\xi_{k}\right)^{-s_{0}} \in \ell_{\min \{p, q\}}$. By Proposition 5.7, we obtain the embeddings

$$
M_{p, \infty}^{s+s_{0}}(h, w) \hookrightarrow M_{p, q}^{s}(h, w) \hookrightarrow M_{p, \infty}^{s}(h, w) .
$$

and

$$
M_{p, \infty}^{s+s_{0}}(h, w) \hookrightarrow F_{p, q}^{s}(h, w) \hookrightarrow M_{p, \infty}^{s}(h, w) .
$$

Thus, is suffices to show that $\mathcal{S}\left(\mathbb{R}^{d}\right) \hookrightarrow M_{p, \infty}^{s}(h, w) \hookrightarrow \mathcal{S}^{\prime}\left(\mathbb{R}^{d}\right)$. Let

$$
p_{N}(g):=\sup _{\xi} w(\xi)^{N / \kappa} \sum_{|\beta| \leq N}\left|\partial^{\beta} \hat{g}(\xi)\right|, \quad N \in \mathbb{N}, g \in \mathcal{S}\left(\mathbb{R}^{d}\right),
$$

be semi-norms on $\mathcal{S}\left(\mathbb{R}^{d}\right)$. It follows from the growth condition (5.1) that the semi-norms $\left\{p_{N}\right\}$ define the usual topology on $\mathcal{S}\left(\mathbb{R}^{d}\right)$. Take $f \in \mathcal{S}\left(\mathbb{R}^{d}\right)$, and let $K>d / p$. Notice that for $r \in \mathbb{R}$,

$$
\begin{aligned}
\left\|\mathcal{F}^{-1} \varphi_{k} \hat{f}\right\|_{L_{p}} \\
\quad \leq C_{1}\left\|(1+|\cdot|)^{K} \mathcal{F}^{-1} \varphi_{k} \hat{f}\right\|_{L_{\infty}} \leq C_{2} \sum_{|\beta| \leq K}\left\|\mathcal{F}^{-1} \partial^{\beta}\left[\varphi_{k} \hat{f}\right]\right\|_{L_{\infty}} \\
\quad \leq C_{3} \sum_{|\beta| \leq K}\left\|\partial^{\beta}\left[\varphi_{k} \hat{f}\right]\right\|_{L_{1}} \leq C_{4} \sup _{\xi} w(\xi)^{(d+1) / \kappa} \sum_{|\beta| \leq K}\left|\partial^{\beta}\left[\varphi_{k} \hat{f}\right](\xi)\right| \\
\left.\quad \leq C_{5}\left(\sup _{\xi} w(\xi)^{((d+1) / \kappa+r)} \sum_{|\beta| \leq K}\left|\partial^{\beta} \hat{f}(\xi)\right|\right) \times w(\xi)^{-r} \sum_{|\beta| \leq K}\left|\partial^{\beta} \varphi_{k}(\xi)\right|\right) \\
\quad \leq C_{6} w\left(\xi_{k}\right)^{-r} p_{N}(f),
\end{aligned}
$$

for $N \geq \max (K,(d+1) / \kappa+r)$, where we have used that $w$ is a moderate weight in the last estimate. Thus $\|f\|_{M_{p, \infty}^{r}(h, w)} \leq C p_{N}(f)$ for $N>$ $\max (d / p,(d+1) / \kappa+r)$, so $\mathcal{S}\left(\mathbb{R}^{d}\right) \hookrightarrow M_{p, \infty}^{s}(h, w)$. Also notice that for $s \in \mathbb{R}$ we can pick $r$ such that $r-s>s_{0} \min \{p, q\}$ to obtain

$$
\sum_{k} w\left(\xi_{k}\right)^{s}\left\|\mathcal{F}^{-1} \varphi_{k} \hat{f}\right\|_{L_{p}} \leq C \sum_{k} w\left(\xi_{k}\right)^{-r+s} p_{N}(f) \leq C^{\prime} p_{N}(f),
$$

so $\mathcal{S}\left(\mathbb{R}^{d}\right) \hookrightarrow M_{1,1}^{s}(h, w)$. We now prove that $M_{p, \infty}^{s}(h, w) \hookrightarrow \mathcal{S}^{\prime}\left(\mathbb{R}^{d}\right)$. For $f \in M_{p, \infty}^{s}(h, w)$ and $\theta \in \mathcal{S}\left(\mathbb{R}^{d}\right)$ we have (formally)

$$
\langle f, \theta\rangle=\langle\hat{f}, \hat{\theta}\rangle=\sum_{k}\left\langle\varphi_{k} \hat{f}, \hat{\theta}\right\rangle=\sum_{k}\left\langle\varphi_{k} \hat{f}, \varphi_{k}^{*} \hat{\theta}\right\rangle=\sum_{k}\left\langle\mathcal{F}^{-1}\left(\varphi_{k} \hat{f}\right), \mathcal{F}^{-1}\left(\varphi_{k}^{*} \hat{\theta}\right)\right\rangle .
$$


Using the Nikol'skij-Plancherel-Polya inequality [36, Proposition 1.3.2], and that $\operatorname{supp}\left(\varphi_{k}\right)$ is contained in the Euclidean ball $B\left(\xi_{k}, \rho^{\prime} h\left(\xi_{k}\right)\right)$, yields

$$
\begin{aligned}
|\langle f, \theta\rangle| & \leq \sum_{k}\left\|\mathcal{F}^{-1}\left(\varphi_{k} \hat{f}\right)\right\|_{L_{\infty}}\left\|\mathcal{F}^{-1}\left(\varphi_{k}^{*} \hat{\theta}\right)\right\|_{L_{1}} \\
& \leq c \sum_{k} h\left(\xi_{k}\right)^{d / p}\left\|\mathcal{F}^{-1}\left(\varphi_{k} \hat{f}\right)\right\|_{L_{p}}\left\|\mathcal{F}^{-1}\left(\varphi_{k}^{*} \hat{\theta}\right)\right\|_{L_{1}} \\
& \leq c^{\prime}\|f\|_{M_{p, \infty}^{s}(h, w)} \sum_{k} w\left(\xi_{k}\right)^{-s} h\left(\xi_{k}\right)^{d / p}\left\|\mathcal{F}^{-1}\left(\varphi_{k}^{*} \hat{\theta}\right)\right\|_{L_{1}} \\
& \leq C c^{\prime}\|f\|_{M_{p, \infty}^{s}(h, w)} \sum_{k} w\left(\xi_{k}\right)^{-s} w\left(\xi_{k}\right)^{\alpha_{2} d /(\kappa p)}\left\|\mathcal{F}^{-1}\left(\varphi_{k}^{*} \hat{\theta}\right)\right\|_{L_{1}}, \\
& \leq C c^{\prime \prime}\|f\|_{M_{p, \infty}^{s}(h, w)}\|\theta\|_{M_{1,1}^{-s+\alpha_{2} d /(\kappa p)}(h, w)} \cdot \operatorname{since} h(\xi) \leq C w(\xi)^{\alpha_{2} / \kappa},
\end{aligned}
$$

Finally, since $\mathcal{S}\left(\mathbb{R}^{d}\right) \hookrightarrow M_{1,1}^{-s+\alpha_{2} d /(\kappa p)}(h, w)$, we obtain $|\langle f, \theta\rangle| \leq$ $C\|f\|_{M_{p, \infty}^{s}(h, w)} p_{N}(\theta)$ for sufficiently large $N$ (with $N$ independent of $f$ ). We conclude that $M_{p, \infty}^{s}(h, w) \hookrightarrow \mathcal{S}^{\prime}\left(\mathbb{R}^{d}\right)$.

Let us now show that $F_{p, q}^{s}(h, w)$ is complete. Let $\left\{f_{n}\right\}_{n \in \mathbb{N}}$ be a Cauchysequence in $F_{p, q}^{s}(h, w)$. Then $\left\{f_{n}\right\}_{n \in \mathbb{N}}$ is a Cauchy-sequence in $\mathcal{S}^{\prime}\left(\mathbb{R}^{d}\right)$, and since $\mathcal{S}^{\prime}\left(\mathbb{R}^{d}\right)$ is complete, $f_{n}$ converges in $\mathcal{S}^{\prime}\left(\mathbb{R}^{d}\right)$ to an element $f \in \mathcal{S}^{\prime}\left(\mathbb{R}^{d}\right)$. It follows easily that $\left(\mathcal{F}^{-1} \varphi_{k} \hat{f}_{n}\right)(x) \rightarrow\left(\mathcal{F}^{-1} \varphi_{k} \hat{f}\right)(x)$ pointwise as $n \rightarrow \infty$. We apply Fatou's lemma twice to obtain (for $q<\infty$ )

$$
\begin{aligned}
\left\|f-f_{n}\right\|_{F_{p, q}^{s}(h, w)} & =\left\|\left(\sum_{k} w\left(\xi_{k}\right)^{q s} \varphi_{k}(D)\left(f-f_{n}\right)^{q}\right)^{1 / q}\right\|_{L_{p}} \\
& \leq\left\|\liminf _{m \rightarrow \infty}\left(\sum_{k} w\left(\xi_{k}\right)^{q s} \varphi_{k}(D)\left(f_{m}-f_{n}\right)^{q}\right)^{1 / q}\right\|_{L_{p}} \\
& \leq \liminf _{m \rightarrow \infty}\left\|\left(\sum_{k} w\left(\xi_{k}\right)^{q s} \varphi_{k}(D)\left(f_{m}-f_{n}\right)^{q}\right)^{1 / q}\right\|_{L_{p}} \\
& =\liminf _{m \rightarrow \infty}\left\|f_{m}-f_{n}\right\|_{F_{p, q}^{s}(h, w)},
\end{aligned}
$$

which shows that $f \in F_{p, q}^{s}(h, w)$ and $\left\|f-f_{n}\right\|_{F_{p, q}^{s}(h, w)} \rightarrow 0$. Similar arguments can be used to show that $M_{p, q}^{s}(h, w)$ is complete.

To prove that the Schwartz space $\mathcal{S}\left(\mathbb{R}^{d}\right)$ is dense in $F_{p, q}^{s}(h, w)$, we first approximate $f \in F_{p, q}^{s}(h, w)$ by a bandlimited function $\tilde{f}$. Then we obtain a Schwartz class approximation to $\tilde{f}$ using a (compactly supported) mollifier. For further details, we refer to the proof of Theorem 2.3.3 in [36]. The same result holds true for $M_{p, q}^{s}(h, w)$, provided $p, q<\infty$. 
We conclude this appendix by proving the following technical lemma which is used to prove Theorem 6.4. We use the same notation as in Section 6 .

Lemma A.1. Let $0<r \leq 1$. There exists a constant $C$ such that for any sequence $\left\{s_{k, n}\right\}_{k, n}$ we have

$$
\sum_{n}\left|s_{k, n}\right|\left|\eta_{k, n}\right| \leq C\left|T_{k}\right|^{1 / 2} M_{r}^{B}\left(\sum_{n}\left|s_{k, n}\right| \chi_{Q(k, n)}\right) .
$$

Proof. From (4.5) we have that $\left|\eta_{k, n}(x)\right| \leq C_{N}\left|T_{k}\right|^{1 / 2}\left(1+\mid \frac{\pi}{a} n+\right.$ $\left.\left.\delta_{t_{k}}^{\top} x\right|_{B}\right)^{-N}$ for any $N>0$. Fix $N>\nu / r$. We can, without loss of generality, suppose $x \in Q(k, 0)$. Let $A_{0}=\left\{n \in \mathbb{Z}^{d}:\left|\frac{\pi}{a} n\right|_{B} \leq 1\right\}$, and for $j \in \mathbb{N}$, let $A_{j}=\left\{n \in \mathbb{Z}^{d}: 2^{j-1}<\left|\frac{\pi}{a} n\right|_{B} \leq 2^{j}\right\}$. Notice that $\cup_{n \in A_{j}} Q(k, n)$ is a bounded set contained in the ball $\mathcal{B}_{B}\left(0, c 2^{j+1} t_{k}^{-1}\right)$. Now,

$$
\begin{aligned}
& \sum_{n \in A_{j}}\left|s_{k, n}\right|\left(1+\left|\frac{\pi}{a} n+\delta_{t_{k}}^{\top} x\right|_{B}\right)^{-N} \\
& \leq C 2^{-j N} \sum_{n \in A_{j}}\left|s_{k, n}\right| \\
& \leq C 2^{-j N}\left(\sum_{n \in A_{j}}\left|s_{k, n}\right|^{r}\right)^{1 / r} \\
& \leq C 2^{-j N} t_{k}^{\nu / r}\left(\int \sum_{n \in A_{j}}\left|s_{k, n}\right|^{r} \chi_{Q(k, n)}(y) d y\right)^{1 / r} \\
& \leq C L^{1-r} 2^{-j N} t_{k}^{\nu / r}\left(\int_{\mathcal{B}_{B}\left(0, c 2^{j+1} t_{k}^{-1}\right)}\left(\sum_{n \in A_{j}}\left|s_{k, n}\right| \chi_{Q(k, n)}(y)\right)^{r} d y\right)^{1 / r} \\
& \leq C^{\prime} 2^{-j(N-\nu / r)} M_{r}^{B}\left(\sum_{n \in \mathbb{Z}^{d}}\left|s_{k, n}\right| \chi_{Q(k, n)}\right)(x) .
\end{aligned}
$$

The result now follows by summing over $j \in \mathbb{N}_{0}$.

\section{References}

[1] R. Beals and C. Fefferman, Classes of spatially inhomogeneous pseudodifferential operators, Proc. Nat. Acad. Sci. U.S.A., 70(1973), 1500-1501.

[2] O. V. Besov, Investigation of a class of function spaces in connection with imbedding and extension theorems, Trudy. Mat. Inst. Steklov., 60(1961), 42-81. 
[3] L. Borup, Pseudodifferential operators on $\alpha$-modulation spaces, J. Funct. Spaces Appl., 2(2)(2004), 107-123.

[4] L. Borup and M. Nielsen, Boundedness for pseudodifferential operators on multivariate $\alpha$-modulation spaces, Ark. Mat., 44(2)(2006), 241-259.

[5] L. Borup and M. Nielsen, Nonlinear approximation in $\alpha$-modulation spaces, Math. Nachr., 279(1-2)(2006), 101-120.

[6] L. Borup and M. Nielsen, Frame decomposition of decomposition spaces, J. Fourier Anal. Appl., 13(1)(2007), 39-70.

[7] G. Bourdaud, $L^{p}$ estimates for certain nonregular pseudodifferential operators, Comm. Partial Differential Equations, 7(9)(1982), 10231033.

[8] M. Bownik, Atomic and molecular decompositions of anisotropic Besov spaces, Math. Z., 250(3)(2005) 539-571.

[9] M. Bownik and K.-P. Ho, Atomic and molecular decompositions of anisotropic Triebel-Lizorkin spaces, Trans. Amer. Math. Soc., 358 (4) (2006), 1469-1510.

[10] H. Q. Bui, On Besov, Hardy and Triebel spaces for $0<p \leq 1$, Ark. Mat., 21(2)(1983)169-184.

[11] A.-P. Calderón and A. Torchinsky, Parabolic maximal functions associated with a distribution, Advances in Math., 16(1975), 1-64.

[12] A. Córdoba and C. Fefferman, Wave packets and Fourier integral operators, Comm. Partial Differential Equations 3(11)(1978), 9791005.

[13] W. Czaja, Boundedness of pseudodifferential operators on modulation spaces, J. Math. Anal. Appl., 284(1)(2003), 389-396.

[14] H. G. Feichtinger, Banach spaces of distributions defined by decomposition methods II, Math. Nachr., 132(1987), 207-237.

[15] H. G. Feichtinger, Amalgam spaces and generalized harmonic analysis, In: Proceedings of the Norbert Wiener Centenary Congress, 1994 (East Lansing, MI, 1994), Volume 52 of Proc. Sympos. Appl. Math. pages 141-150, Providence, RI, 1997. Amer. Math. Soc.

[16] H. G. Feichtinger, Modulation spaces of locally compact abelian groups In: Proc. Internat. Conf. on Wavelets and Applications, pages 1-56. Allied Publishers, New Delhi, 2003.

[17] H. G. Feichtinger and P. Gröbner, Banach spaces of distributions defined by decomposition methods. I, Math. Nachr., 123(1985), 97-120.

[18] H. G. Feichtinger and K. Gröchenig, A unified approach to atomic decompositions via integrable group representations, In: Function 
spaces and applications (Lund, 1986), Volume 1302 of Lecture Notes in Math. pages 52-73. Springer, Berlin, 1988.

[19] H. G. Feichtinger and K. H. Gröchenig, Banach spaces related to integrable group representations and their atomic decompositions. I, J. Funct. Anal., 86(2)(1989), 307-340.

[20] H. G. Feichtinger and K. H. Gröchenig, Banach spaces related to integrable group representations and their atomic decompositions. II, Monatsh. Math., 108(2-3)(1989), 129-148.

[21] M. Frazier and B. Jawerth, Decomposition of Besov spaces, Indiana Univ. Math., J. 34(4)(1985), 777-799.

[22] M. Frazier and B. Jawerth, A discrete transform and decompositions of distribution spaces, J. Funct. Anal., 93(1)(1990), 34-170.

[23] G. Gibbons, Opérateurs pseudo-différentiels et espaces de Besov, C. R. Acad. Sci. Paris Sér. A-B, 286(20)(1978), A895-A897.

[24] P. Gröbner, Banachräume glatter Funktionen und Zerlegungsmethoden, $\mathrm{PhD}$ thesis, University of Vienna, 1992.

[25] K. Gröchenig, Describing functions: atomic decompositions versus frames, Monatsh. Math., 112(1)(1991), 1-42.

[26] K. Gröchenig and C. Heil, Modulation spaces and pseudodifferential operators, Integral Equations Operator Theory 34(4)(1999), 439-457.

[27] H. Kumano-go, Pseudodifferential operators, MIT Press, Cambridge, Mass., 1981 Translated from the Japanese by the author, Rémi Vaillancourt and Michihiro Nagase.

[28] B. Nazaret and M. Holschneider, An interpolation family between Gabor and wavelet transformations: application to differential calculus and construction of anisotropic Banach spaces, In: Nonlinear hyperbolic equations, spectral theory, and wavelet transformations, volume 145 of Oper. Theory Adv. Appl. pages 363-394. Birkhäuser, Basel, 2003.

[29] L. Päivärinta, Pseudodifferential operators in Hardy-Triebel spaces, Z. Anal. Anwendungen 2(3)(1983), 235-242.

[30] L. Päivärinta and E. Somersalo, A generalization of the CalderónVaillancourt theorem to $L^{p}$ and $h^{p}$, Math. Nachr., 138(1988), 145-156.

[31] S. Pilipović and N. Teofanov, Pseudodifferential operators on ultramodulation spaces, J. Funct. Anal., 208(1)(2004), 194-228. 
[32] E. M. Stein, Harmonic analysis: real-variable methods, orthogonality, and oscillatory integrals, Volume 43 of Princeton Mathematical Series Princeton University Press, Princeton, NJ, 1993, With the assistance of Timothy S. Murphy, Monographs in Harmonic Analysis, III.

[33] E. M. Stein and S. Wainger, Problems in harmonic analysis related to curvature, Bull. Amer. Math. Soc., 84(6)(1978), 1239-1295.

[34] K. Tachizawa, The boundedness of pseudodifferential operators on modulation spaces, Math. Nachr., 168(1994), 263-277.

[35] J. Toft, Continuity properties for modulation spaces, with applications to pseudo-differential calculus. I, J. Funct. Anal., 207(2)(2004), 399429.

[36] H. Triebel, Theory of Function Spaces, Birkhäuser Verlag, Basel, 1983.

[37] H. Triebel, Theory of Function Spaces. II, Birkhäuser Verlag, Basel, 1992.

[38] H. Triebel, Interpolation Theory, Function Spaces, Differential Operators, Johann Ambrosius Barth, Heidelberg, 1995.

[39] M. Yamazaki, A quasihomogeneous version of paradifferential operators. I, Boundedness on spaces of Besov type, J. Fac. Sci. Univ. Tokyo Sect. IA Math., 33(1)(1986), 131-174.

[40] M. Yamazaki, A quasihomogeneous version of paradifferential operators. II. A symbol calculus, J. Fac. Sci. Univ. Tokyo Sect. IA Math, 33(2)(1986), 311-345.

Department of Mathematical Sciences

Aalborg University

Fredrik Bajers Vej 7G

DK-9220 Aalborg East

Denmark

(E-mail : lasse@math.aau.dk)

(E-mail : mnielsen@math.aau.dk)

(Received : May 2007) 


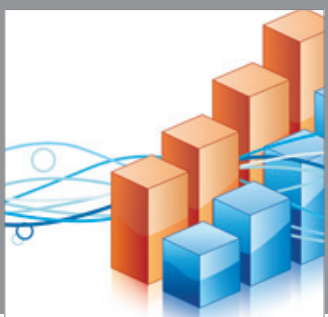

Advances in

Operations Research

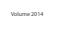

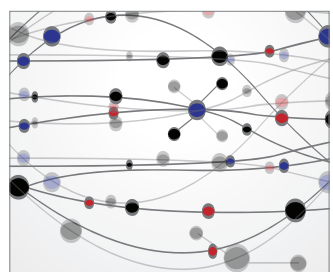

\section{The Scientific} World Journal
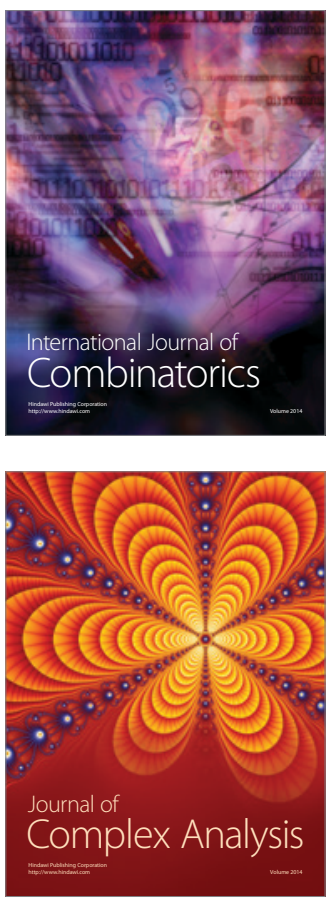

International Journal of

Mathematics and

Mathematical

Sciences
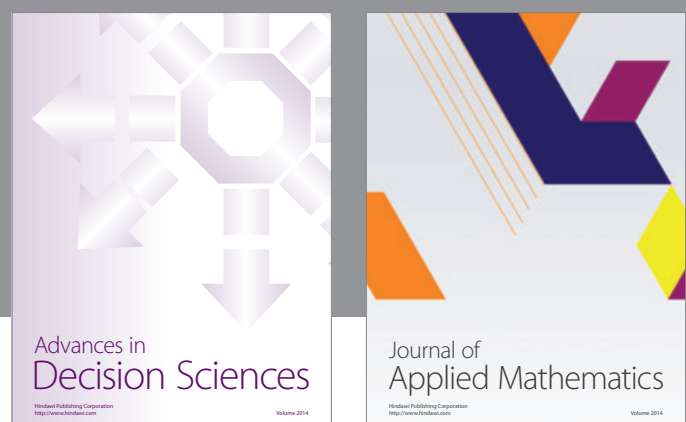

Journal of

Applied Mathematics
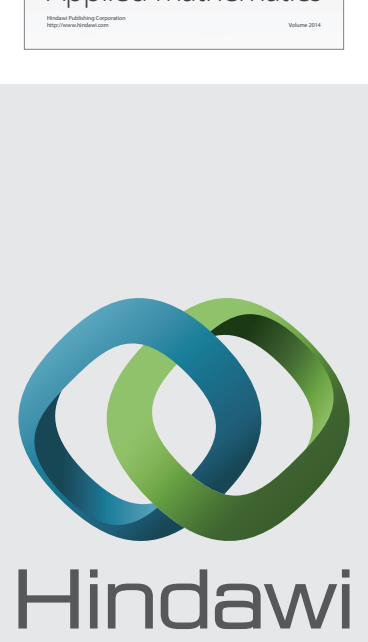

Submit your manuscripts at http://www.hindawi.com
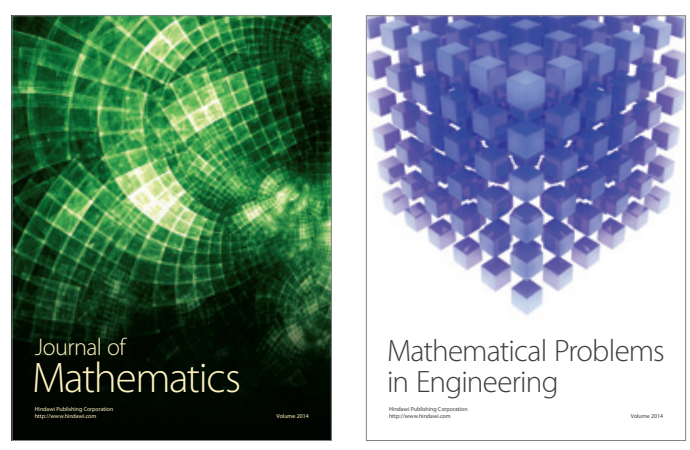

Mathematical Problems in Engineering
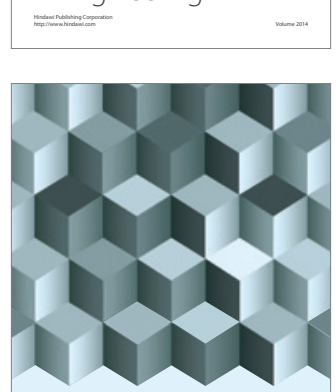

Journal of

Function Spaces
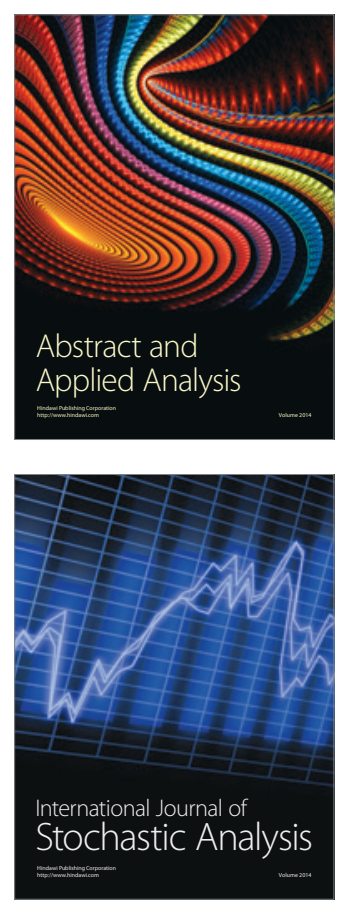

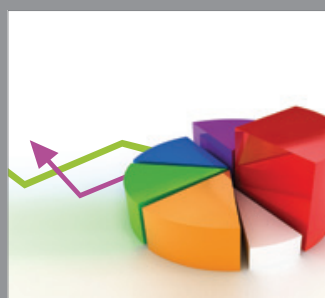

ournal of

Probability and Statistics

Promensencen
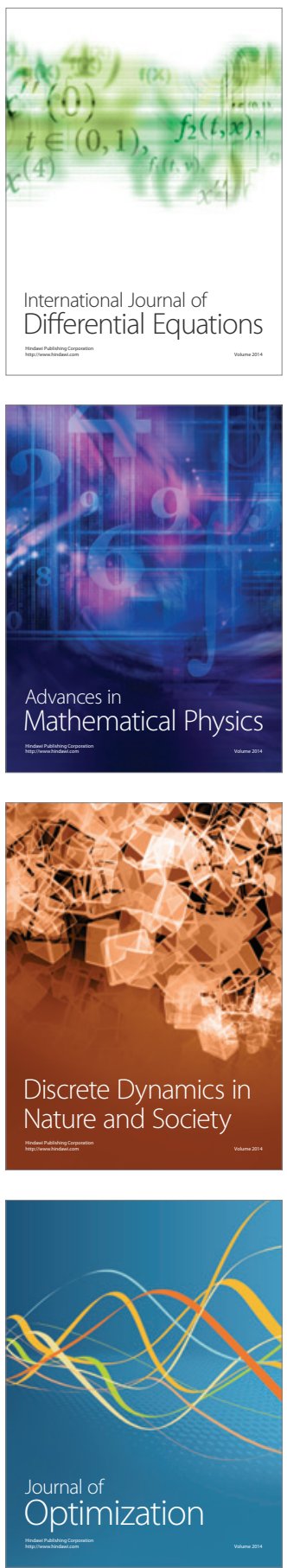